\title{
Archaeonautica
}

Archaeonautica

L'archéologie maritime et navale de la préhistoire à

l'époque contemporaine

19 | 2017

L'archéologie maritime et navale de la préhistoire à

l'époque contemporaine

\section{Les différents ports du delta du Rhône au Haut Empire : modèle économique autour de la circulation et la diffusion des produits}

The different ports of the Rhone delta in the Early Empire: an economic model for the traffic and distribution of goods

\section{David Djaoui}

\section{OpenEdition} Journals

\section{Édition électronique}

URL : http://journals.openedition.org/archaeonautica/496

DOI : 10.4000/archaeonautica.496

ISSN : 2117-6973

Éditeur

CNRS Éditions

\section{Édition imprimée}

Date de publication : 7 décembre 2017

Pagination : 123-140

ISBN : 978-2-271-11766-3

ISSN : 0154-1854

Référence électronique

David Djaoui, « Les différents ports du delta du Rhône au Haut Empire : modèle économique autour de la circulation et la diffusion des produits », Archaeonautica [En ligne], 19 | 2017, mis en ligne le 30 avril 2020, consulté le 30 avril 2020. URL : http://journals.openedition.org/archaeonautica/496 ; DOI : https://doi.org/10.4000/archaeonautica.496 


\title{
LES DIFFÉRENTS PORTS DU DELTA DU RHÔNE AU HAUT EMPIRE: MODĖLE ÉCONOMIQUE AUTOUR DE LA CIRCULATION ET LA DIFFUSION DES PRODUITS
}

\author{
David DJAOUI
}

\begin{abstract}
"It is becoming increasingly clear that ports cannot be viewed in isolation, or simply in relation to the sea. They occupy liminal positions between land and sea that can be appreciated only by looking at their relationships to surrounding hinterlands and to other ports."
\end{abstract}

Simon Keay, The Port system of imperial Rome, London, 2012.

\begin{abstract}
Résumé
Les recherches menées par le DRASSM au large des Saintes-Mariesde-la-Mer ont révélé une concentration exceptionnelle d'épaves située face à un ancien bras du Rhône, plus connu sous le vocable médiéval de Rhône Saint-Ferréol. Située face à cette embouchure, la mise en évidence d'une zone de mouillage associée à des structures portuaires permettait de défendre l'idée que le «complexe» des Saintes-Mariesde-la-Mer constituait le seul véritable avant-port d'Arles. Avec la présence d'au moins trente épaves chargées de matières premières, j'ai développé l'hypothèse que le Rhône Saint-Ferréol était dévolu aux matières premières et que les navires chargés d'amphores, situés loin de la côte, devaient croiser au large pour éviter la Camargue. Cette réflexion a conduit à envisager que les autres bras du Rhône, que ce soit celui de Daladel et/ou celui d'Ulmet, devaient être non seulement navigables mais également réservés aux amphores.

Cet article présente une première synthèse pour tenter de définir les différents ports potentiels qui pourraient assurer une circulation spécialisée des marchandises. L'intégration de l'ensemble de ces critères permettra de proposer un modèle économique à l'échelle du delta du Rhône selon lequel aussi bien Arles, les Saintes-Maries-de-la-Mer, le golfe de Fos, I'anse Saint-Gervais que les autres embouchures du Rhône ne constitueraient non plus des «avant-ports» mais de véritables ports.
\end{abstract}

Mots clefs: Port, espace fluvio-maritime, navigation, épave, commerce, amphore, matières premières, Arles, Haut-Empire, Rhône, Fossur-Mer, delta du Rhône, archéologie navale

\begin{abstract}
The different ports of the Rhone delta in the Early Empire: an economic model for the traffic and distribution of goods

Research conducted by the DRASSM off Les Saintes-Maries-de-la-Mer have revealed an exceptional concentration of shipwrecks located in front of an ancient branch of the Rhone known by the medieval term of Rhone Saint-Ferréol. The discovery of a mooring area associated with harbour structures off this mouth supported the idea that the "complex" of Les Saintes-Maries-de-la-Mer was the only true outport of Arles. The presence of at least thirty wrecks loaded with raw materials promoted the theory that Rhone Saint-Ferréol was dedicated to raw materials and that ships loaded with amphora, located far from the coastline, must have navigated further out to avoid the Camargue. This has led to the notion that the other arms of the Rhone, whether that of Daladel and/or Ulmet were not only navigable but also reserved for amphorae.

This paper will present an initial synthesis that attempts to define the different potential harbours that might have serviced a specialised traffic in merchandise. The integration of the ensemble of criteria will lead to the proposition of an economic model on the scale of the Rhone delta, according to which Arles, Les Saintes-Maries-de-la-Mer, Fos, Saint-Gervais Bay and the other mouths of the Rhone were not simply "outports" but true ports.
\end{abstract}

Key words: Harbor, fluviomaritime area, navigation, shipwreck, trade, amphora, raw materials, Arles, Early Empire, Rhône, Fos-sur-Mer, Rhone delta, naval architecture
Depuis plus d'une trentaine d'années, les différentes campagnes de carte archéologique dirigées par Luc Long (Drassm/MCC) ${ }^{1}$, ont permis d'inventorier trente-neuf épaves antiques en Camargue. Localisées entre l'Espiguette et l'ouest des Saintes-Maries-dela-Mer (Fig. 1), ces différentes épaves peuvent se scinder en deux groupes (Fig. 2). D'un côté les trente-deux bateaux qui se sont échoués sur les lobes alluviaux en essayant de remonter le Rhône Saint-Ferréol et qui se situent aujourd'hui, entre 12 et $14 \mathrm{~m}$ de profondeur, soit à $2 \mathrm{~km}$ du rivage actuel. Et de l'autre côté, les épaves dites «profondes » qui rassemblent sept gisements amphoriques et dont l'éloignement à la côte, qui peut atteindre les 40 milles

1. Comptage réalisé à partir de Long 1997, Long, Illouze 2004 et des nouvelles découvertes ( $C f$. http://2asm-rhone-cesar.blogspot.fr/). nautiques (épave Plages d'Arles 4 échouée par $662 \mathrm{~m}$ de profondeur soit environ à $74 \mathrm{~km}$ de ladite plage), implique non seulement des conditions de naufrages différents mais également un cap que l'on peut qualifier d'«indéterminé».

Pour le Haut-Empire, ces deux groupes d'épaves se différencient également par la nature des cargaisons transportées. Le premier groupe d'épaves est situé à proximité de l'embouchure du Rhône Saint-Ferréol et rassemble trente gisements composés uniquement de matières premières brutes ou semi-transformées ${ }^{2}$. Et le deuxième

2. On compte en effet une épave chargée d'étain (SM17), trois de galettes de cuivre (SM7, SM26, SM30), trois de lingots de plomb (SM1, SM12, SM35), une composée d'une cargaison secondaire de bronze recyclé (SM4), cinq de blocs de marbre 


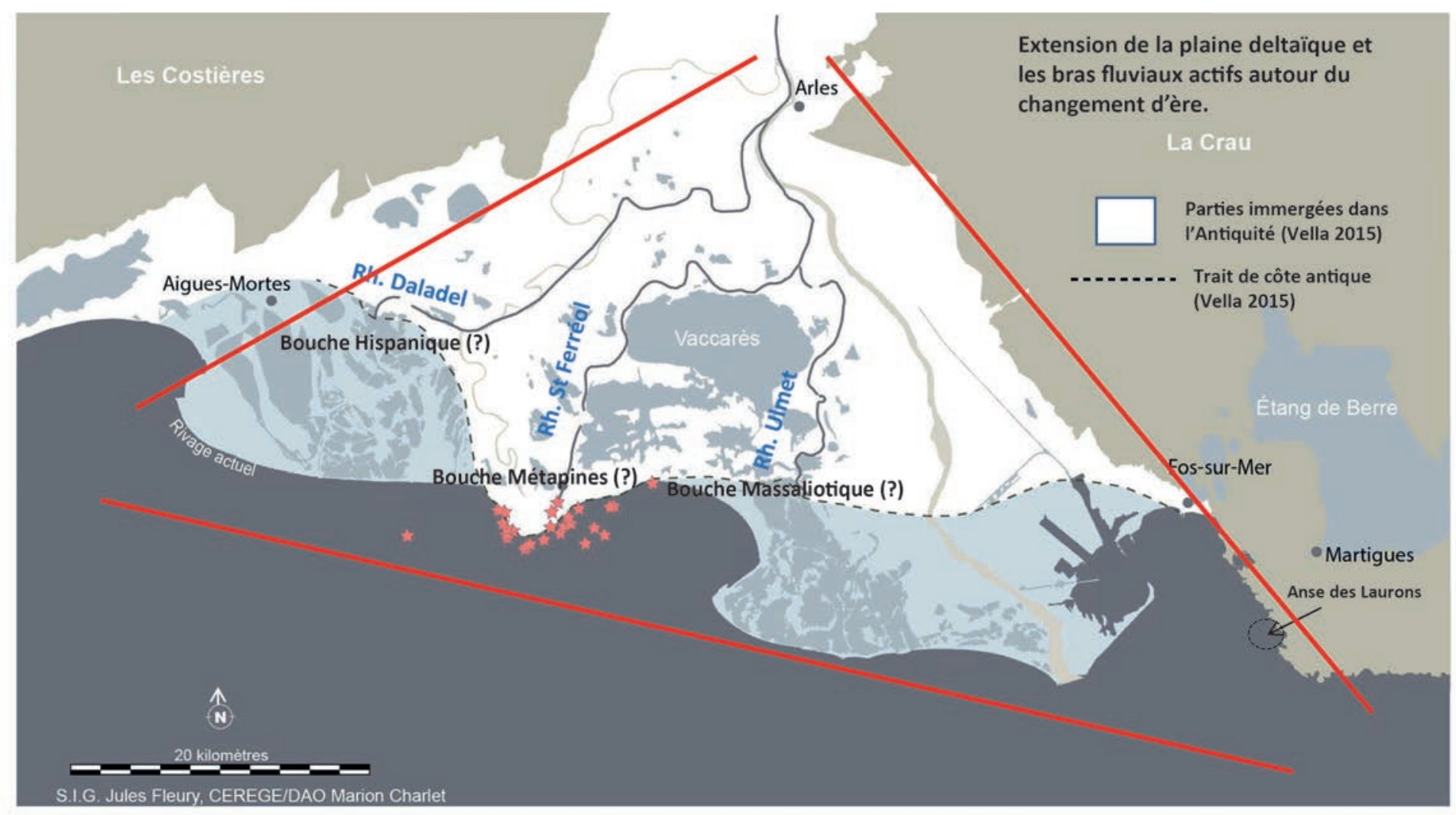

* Epaves antiques des Saintes-Maries-de-la-Mer ( Long 2004)

Fig. 1: Les limites du delta du Rhône (D.A.O.: D. Djaoui).

groupe d'épaves, dites «profondes », qui est éloigné des lobes alluviaux et rassemble quatre épaves chargées d'amphores de Bétique des $\mathrm{I}^{\mathrm{er}}$ et $\mathrm{II}^{\mathrm{e}} \mathrm{s}$. ap. J.-C.

Ce constat m'a conduit à envisager que le Rhône Saint-Ferréol était dévolu à la circulation des matières premières et que les navires chargés d'amphores devaient emprunter l'un des deux autres bras du Rhône, ou encore le canal de Marius (Djaoui à par. 3). Pour le Haut-Empire, les études géomorphologiques s'accordent aussi bien avec les sources écrites (Pline l'Ancien, H.N., III, 5) qu'iconographiques ${ }^{3}$, pour situer en effet deux autres bras naturels, celui de Daladel à l'ouest (l'Ostium Hispaniense $=$ le bras Hispanique) et le Rhône d'Ulmet à l'est (Gradus Massalioticum = la bouche Massaliotique $)^{4}$.

En partant donc du principe que les bateaux chargés d'amphores ne s'orientaient pas en direction du Rhône Saint-Ferréol, de nombreuses questions se posent, tant sur l'exclusivité portuaire des Saintes-Maries-de-la-Mer, que sur son mode de fonctionnement. Quelles sont en effet les données factuelles pour envisager la présence d'un port situé face à l'embouchure du Rhône SaintFerréol? Dispose-t-on dans ce secteur d'une zone de mouillage de qualité pour procéder aux transbordements des amphores et

(SM18, SM21, SM22, SM28, SM39) et enfin 17 épaves chargées de fer (SM2, SM3, SM6, SM8, SM9.1, SM9.2, SM10, SM11, SM13(?), SM23, SM24, SM25, SM27, SM29, SM31, SM32, SM41). À ces différentes matières premières, s'ajoutent également, pour l'une d'entre elle, des rondins de bois en cargaison secondaire (SM24) (Djaoui à par. 3)

3. Mosaïque de la Statio 27 des naviculaires arlésiens d'Ostie du II $^{\mathrm{e}}$ s. et la Table de Peutinger, copie médiévale d'une carte romaine du $\mathrm{III}^{\mathrm{e}}$ siècle.

4. Arnaud-Fassetta 2004; Vella et al. 2005; Vella et al. 2016; Rey et al. 2009; Landuré, et al. (dir.) 2015. des matériaux pondéreux? La présence d'un dépotoir amphorique dans cette zone deltaïque matérialise-t-elle forcément une zone de rupture de charge? Et les alignements de pierres trouvés à proximité du chenal d'entrée du Rhône Saint-Ferréol peuvent-ils être interprétés comme des bases de poteaux supportant des hangars?

Après avoir rejeté l'hypothèse d'un port situé face à l'embouchure du Rhône Saint-Ferréol et préféré celle d'un port situé davantage au large des Saintes-Maries-de-la-Mer, la réflexion d'une spécialisation des bras du Rhône s'articulera autour des autres ports disponibles, et/ou des autres moyens de circulation des produits. En fonction de la nature des cargaisons, comestibles (pour les denrées transportées en amphores) ou non comestibles (pour les matières premières), des types de bateaux (fluviomaritimes et maritimes) et de la qualité de l'ancrage, directement impactée par la profondeur et l'état de la mer, il faudra s'interroger sur les différents ports potentiels pour transborder les marchandises et/ou pour attendre les vents favorables. L'intégration de l'ensemble de ces critères permettra de proposer un modèle économique à l'échelle du delta du Rhône au sein duquel aussi bien Arles, les Saintes-Mariesde-la-Mer, le golfe de Fos, l'anse Saint-Gervais et également les autres embouchures du Rhône ne constitueront non plus des «avantports » mais de véritables ports ${ }^{5}$.

5. On peut souligner que cette activité pouvait concerner un espace privé. L'inscription impériale trouvée à Nîmes en 1873 mentionne en effet un Portus Crindauinus ad ripam fluminis Rhodani (CIL, XII, 3313). 


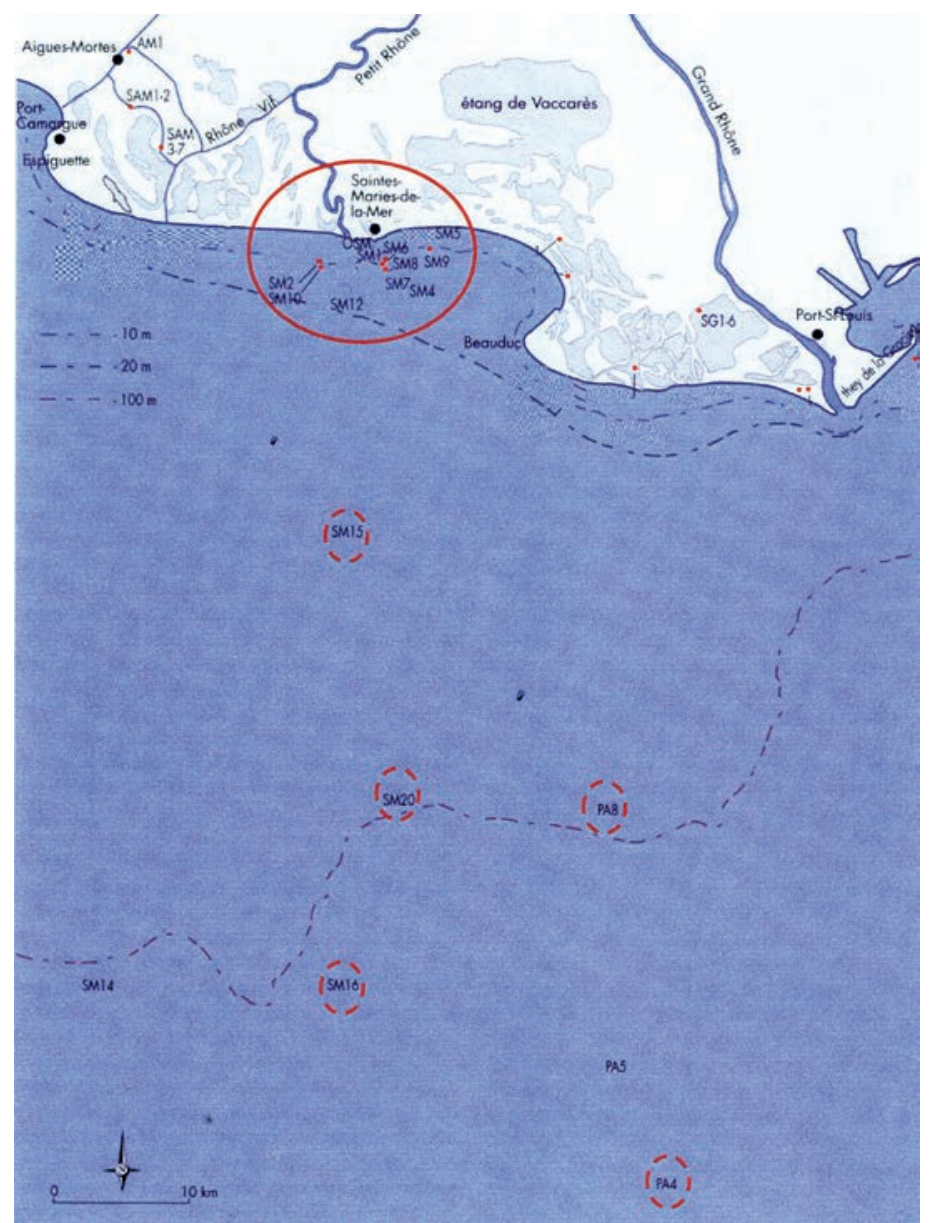

Fig. 2: Contraste entre les épaves antiques situées en bordure du littoral (cercles pleins) et celles, éloignées de la côte, situées en zone profonde (cercles en pointillé) (à partir de L. Long, F. Richez; DAO M. Olive dans Long, Illouze 2004, p. 293, fig. 1).

\section{L'HYPOTHÈSE ACTUELLE: UNE ZONE DE RUPTURE DE CHARGE SITUÉE FACE À L'EMBOUCHURE DU RHÔNE SAINT-FERRÉOL}

Si l'on suit le schéma envisagé par Luc Long, le positionnement des épaves permet de délimiter les contours des barres d'avant-côte sur lesquelles ces bateaux se sont enchâssés (Fig. 3). Aussi, en pointant sur une carte les positions respectives de ces épaves, il reconstitue la forme d'un lobe alluvial qui rappelle, en effet, les relevés cartographiques effectués sur le rivage de Beauduc par les Consuls d'Arles au XVIII ${ }^{\mathrm{e}}$ s. (Fig. 4). L'absence d'épaves entre les lobes, ainsi matérialisés, pourrait suggérer des zones de passages (ou graus) permettant d'atteindre une sorte de «lagon». Protégé des vagues marines par l'émergence de ces formations sédimentaires, cette zone de mouillage naturelle aurait abrité les bateaux maritimes de forts tonnages pour transférer les matériaux les plus pondéreux sur des bateaux de type allège. La découverte d'un grand nombre d'ancres (Fig. 5) et d'innombrables tessons d'amphores constituerait un argument de plus pour délimiter cette zone où «il est loisible d'imaginer que cette vaste rade de mouillage forain servait au transbordement des marchandises depuis les bateaux de mer sur des allèges à fond plat capables de franchir les graus et de remonter le fleuve» (Long, Illouze 2004, p. 324). À proximité de ce dépotoir amphorique, des alignements de blocs calcaires ont été découverts: «Certaines de ces pierres quadrangulaires, qui mesurent jusqu’à
$80 \mathrm{~cm}$ de côté, sont dotées d'un orifice central qui, comme dans l'anse Saint-Gervais, à Fos-sur-Mer, désigne des bases de poteaux » (Long 2008, p. 42). L'alignement de ces blocs, parfois encore liés à du mortier et associés à des clous de charpente et à des fragments de tegulae pourrait matérialiser des hangars ${ }^{6}$. Implantés en milieu lagunaire, la présence de ces magasins de stockage, confortée par la découverte dans la même zone de fragments de dolia, devait permettre de libérer du mouillage les bateaux de mer en période de crue ou d'étiage (Long 2008, p. 42-43). La présence d'un avant-port lié à ces zones de stockage devait induire, selon Luc Long, une activité humaine importante qui pouvait laisser penser à l'existence d'une ancienne agglomération enfouie sous le port actuel de plaisance des Saintes-Maries-de-la-Mer: «Soudain, les noms d'anciennes cités perdues, comme Rhodanousia ou Hérakléia, refont surface» (Long 2008, p. 43).

L'ensemble de ces interprétations permet à l'auteur de défendre l'idée que le «complexe» des Saintes-Maries-de-la-Mer constituerait le seul véritable avant-port d'Arles ${ }^{7}$. Dans ce sens, la mention «duplex Arelate» d'Ausone, c'est-à-dire «Arles la double» (Ausone, Ordo urbilium, 10, v.1), concernerait davantage l'association du port fluvial d'Arles avec les structures portuaires des Saintes-Maries-de-la-Mer (Long 2009, p. 218) plutôt qu'avec celles du port de Fos (Rougé 1987, p. 159-160; Liou, Sciallano 1987, p. 153). En reprenant les différents éléments factuels qui conduisent à cette hypothèse, je procéderai à une lecture critique et proposerai une autre possibilité.

\section{REGARD CRITIQUE SUR CETTE HYPOTHÈSE}

\section{ANALYSE DES ÉLÉMENTS FACTUELS CONCERNANT LA FORMATION D'UNE LAGUNE}

En projetant sur une carte les barres d'avant-côte et les graus, à partir de l'absence et de la présence des épaves, dont la chronologie s'étend sur au moins 150 ans, on fige un système géomorphologique extrêmement complexe pour lequel la mobilité sédimentaire est pourtant perpétuelle (Fig. 3 et 5). De plus, il est délicat d'attester que l'alignement des points des épaves reprend «la forme caractéristique des lobes alluviaux» (Long, Illouze 2004, p. 322). La morphologie de ces derniers décrit en effet une sorte de «patate» informe où seule l'interprétation de l'auteur permet de fixer sa projection. D'un point de vue purement géomorphologique, il est toutefois possible de disposer d'une zone protégée délimitée par des barres d'embouchures émergentes. (Fig. 4). Mais, comme le souligne Claude Vella, ce relief sédimentaire est soumis en permanence aux influences marines et fluviatiles et demeure par conséquent extrêmement fragile. Au moindre coup de tabac, la houle, formée par la mer, peut ainsi déblayer et remanier les matériaux apportés ${ }^{8}$. Il semble par conséquent impossible de projeter un tel mode de fonctionnement à partir du seul positionnement des

6. Long 2009, p. 215-216; Long 2008, p. 42-45; Long, Picard 2009, p. 30-34; Long, Duperron 2011b, p. 98.

7. Long Duperron 2011a, p. 97-98; Long 2009, p. 30-34; Long, Illouze 2004, p. 321-325.

8. Je tiens tout particulièrement à remercier Claude Vella (CEREGE, UMR 7330, Aix-en-Provence) pour la richesse de nos échanges et pour avoir relu une partie de mon article. Pour la barre d'embouchure du Tibre, cette instabilité sédimentaire est bien décrite par Gambarini et Chiesa qui indiquent que les formations de sable peuvent disparaitre en quelques jours quand les vents de Libeccio (sud-ouest) se calment ou immédiatement si l'eau du fleuve remonte (Gambarini, Chiesa 1746, p. 26-27; pour plus de détails voir Boetto 2006, p. 334-335). 


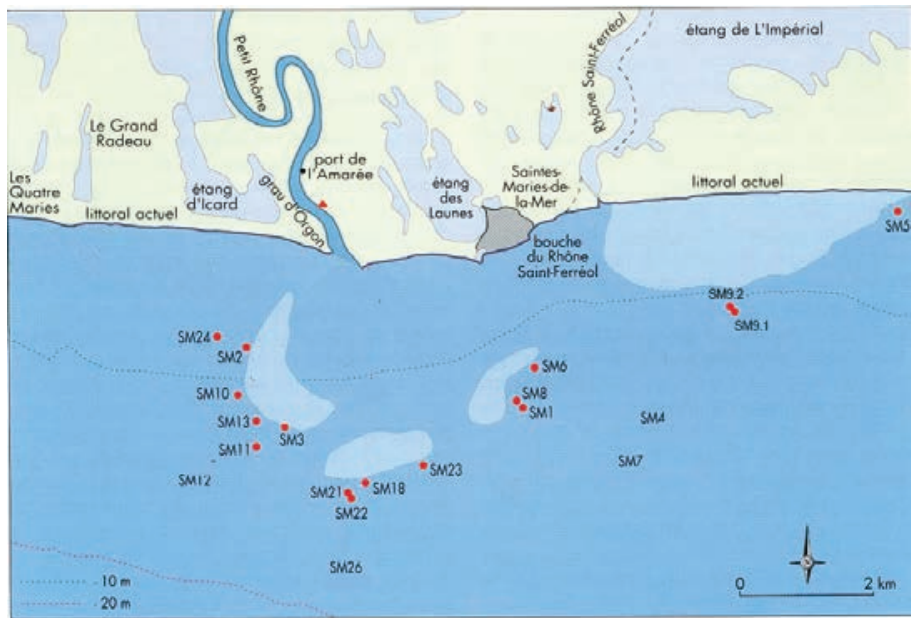

Fig. 3: L'embouchure du Rhône Saint-Ferréol: représentation des lobes alluviaux (en bleu clair) grâce au positionnement des épaves antiques matérialisées par un point rouge (Long, Illouze 2004, p. 323, Fig. 21).

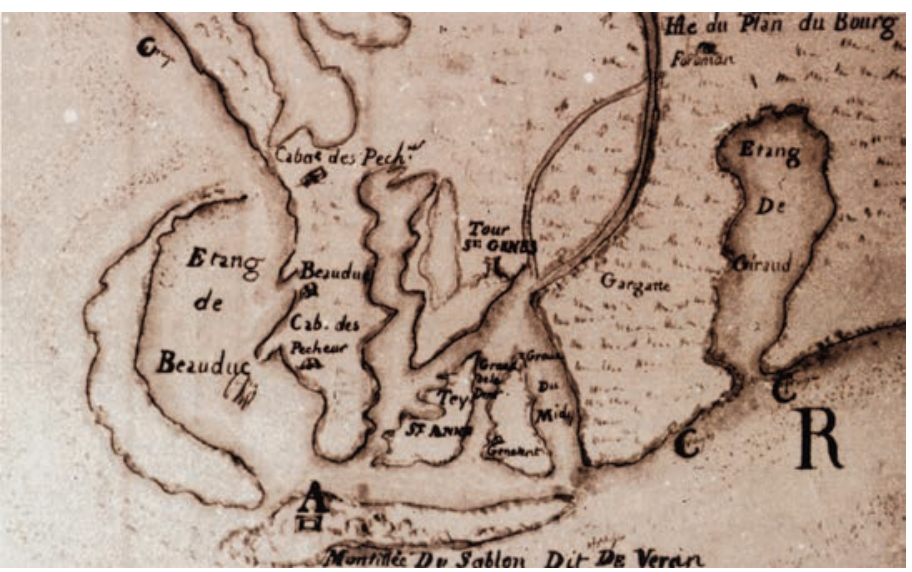

Fig. 4: Carte de l'embouchure du Rhône édifiée par les consuls d'Arles au xvIII siècle: l'Étang de Beauduc est circonscrit par un lobe alluvial émergent de forme semi-circulaire très comparable aux lobes alluviaux dessinés par L. Long à partir des épaves (Long, Illouze 2004, p. 323, Fig. 222; ADBR 200 E 1010; L. Long).

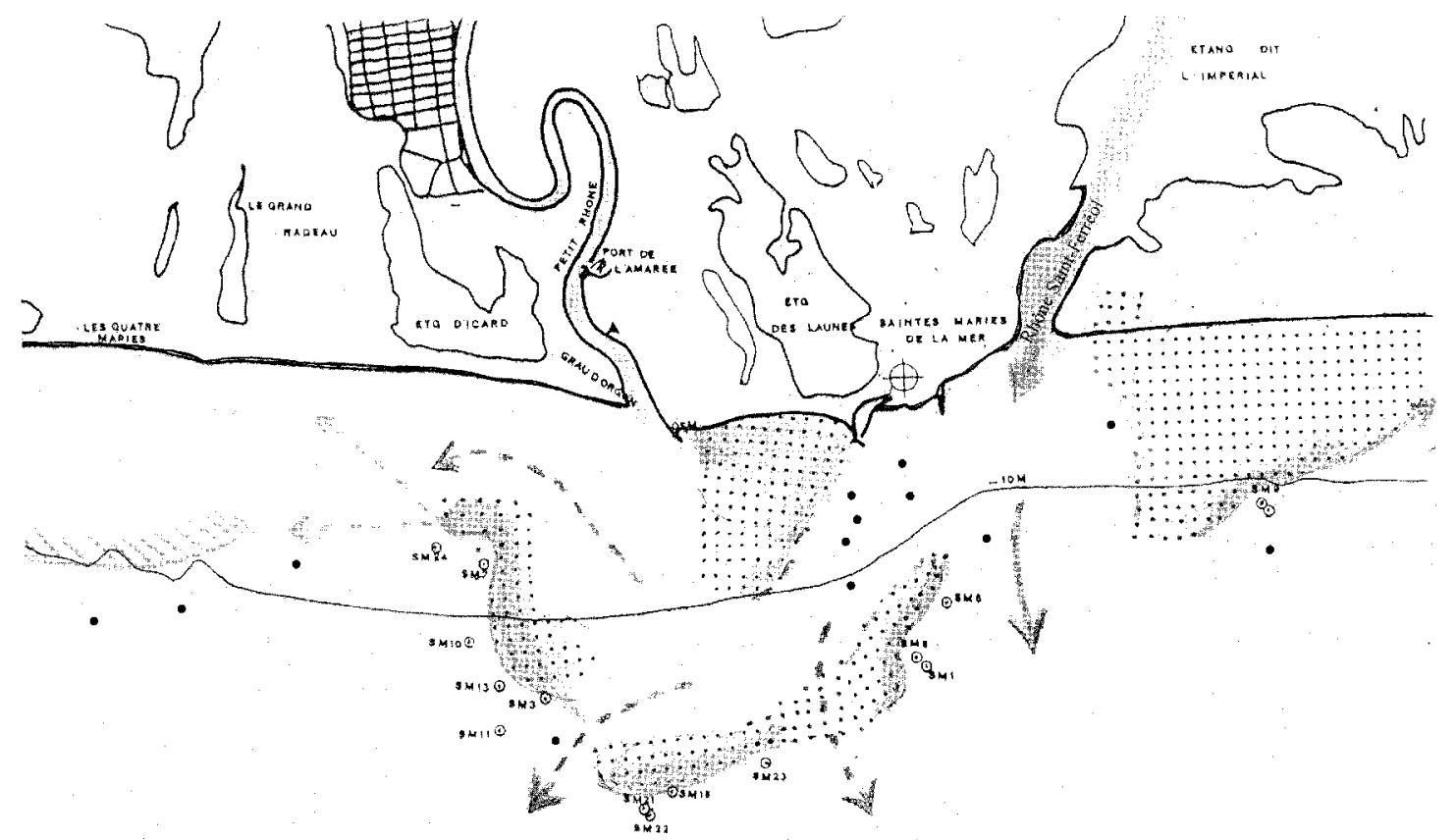

Fig. 5: L'embouchure du Rhône Saint-Ferréol: Représentation plus détaillée qu'offre le positionnement des épaves antiques (rond pointés) et des ancres isolées (ronds noirs) (Long 2003, p. 56, Fig. 44).

épaves. De plus, quand on considère qu' «un navire est d'autant plus en sécurité qu'il est plus apte à prendre le large» (Arnaud 2016, p. 141), on imagine assez bien la souricière dans laquelle pouvaient se trouver des bateaux encerclés aussi bien par la côte, les lobes alluviaux, les autres bateaux en stationnement et/ou en cours de transbordement, ainsi que ceux qui s'engageaient dans la montée ou encore dans la descente du fleuve. En l'absence de preuves formelles de ces lobes émergents, la zone située face à l'embouchure du Rhône Saint-Ferréol est-elle toutefois propice à assurer un mouillage?

\section{MISE EN PERSPECTIVE D'UNE ZONE DE MOUILLAGE SITUÉE FACE À UNE EMBOUCHURE}

Au niveau de l'embouchure du Grand Rhône actuel, une couverture bathymétrique ${ }^{9}$ permet de mieux saisir l'impossibilité géomorphologique de disposer d'un tel «port». Dans cette zone deltaïque, présentant une côte basse et sableuse ${ }^{10}$, toutes les parties blanches

9. Cartographie fournie gracieusement par François Sabatier, Aix-Marseille Université (Sabatier, Kulling, à par.).

10. Pour le $\mathrm{XVII}^{\mathrm{e}}$ s. il faut toutefois signaler dans le delta du Rhône l'existence d'un avant-port côtier situé, non pas face à l'embouchure, mais à l'ouest du Bras de Fer, et séparé de ce dernier par une série d'îles. Il figure sur la carte de Flour de 1638. Mais comme le souligne Patricia Payn-Echalier, en estimant sa durée de vie à un demi-siècle, «rien n'est permanent dans le delta et l'avancée de l'embouchure qui 
de la cartographie constituent des zones très peu profondes inférieures à $2 \mathrm{~m}$ tandis que les parties profondes se situent seulement dans l'axe du chenal, c'est-à-dire dans une zone exposée à un fort courant fluvial (Fig. 6). Autrement dit, un mouillage situé face à l'embouchure du Rhône se trouverait dans des profondeurs comprises entre $2 \mathrm{~m}$ et $4 \mathrm{~m}$ de profondeur seulement ${ }^{11}$. Face aux coups de vent, aussi violents qu'imprévisibles de la Méditerranée, un bateau ancré à si faible profondeur a de fortes chances, en cas de houle, de talonner le fond lorsque la mer se creuse ${ }^{12}$. En présence du vent marin (sud/sud-est), ou du Labech (sud/sud-ouest) le bateau serait porté automatiquement vers la terre avec pour conséquence un très fort risque de naufrage ${ }^{13}$.

Si on peut toujours opposer que le Grand Rhône actuel n'est pas le Rhône Saint-Ferréol, et que le savoir-faire des marins est une donnée empirique qui pouvait répondre à ces difficultés, quelles sont alors les données archéologiques concrètes pour confirmer la présence d'une telle zone de mouillage?

\section{ANALYSE FACTUELLE DE LA PRÉSENCE D'UNE ZONE DE MOUILLAGE}

\section{Examen qualitatif et quantitatif des ancres}

La présence des ancres matérialise-t-elle nécessairement une zone de mouillage?

Sur un inventaire de douze ancres trouvées dans cette zone, sept sont des pierres quadrangulaires de petites dimensions et cinq des jas d'ancre en plomb. Parmi le corpus des ancres en pierre, trois sont de forme ovoïde à trois trous percés (STM.96.S.185, STM.96.S.183 STM.96.S.169), une est munie d'un seul trou dans la partie sommitale (STM.96.S.167), et trois autres portent une simple gorge creusée au centre pour le passage d'une corde (STM.96.S.187, STM.96.S.173; STM.96.S.172). Dans le cas des pierres munies d'un seul trou ou d'une simple gorge, seule la corde assure la liaison avec le bateau et aucun crochet ne permet de se fixer au fond pour stabiliser le bateau ${ }^{14}$. Ces pierres de dimensions modestes, qualifiées d'«ancre-poids», devaient s'utiliser davantage pour lester des filets que pour ancrer des bateaux et peuvent par conséquent être retirées du corpus des ancres. En prenant également en compte les

rendit obsolète la tour du Tampan supprima aussi cet avant-port» (Payn-Echalier 2010, p. 36).

11. Pour Ostie, les entrées sableuses amenées par les crues du Tibre à partir de 165 av. J.-C. et 5 ap. J.-C. diminuent l'épaisseur de la colonne d'eau à $2,5 \mathrm{~m}$ sous le zéro antique. Au début de la période impériale la profondeur du bassin n'est plus que de $60 \mathrm{~cm}$ vers 45 av. J.-C. $/ 25$ ap. J.-C. et seulement $30 \mathrm{~cm}$ entre 80 et 230 ap. J.-C. (Goiran et al. 2016).

12. Talonner: «C'est donner des coups de talon ou toucher sur le fond de la mer ou sur un écueil, par l'arrière de la quille, et avec des secousses plus ou moins fortes, selon l'état de la mer etc.» (Bonnefoux 1855).

13. Comme le souligne justement Pascal Arnaud, les sédiments que dépose le fleuve créent des fonds de bonne tenue et relativement peu profonds qui assurent un bon mouillage, aussi longtemps que la houle n'atteint pas des hauteurs et une puissance incompatibles avec la sécurité du mouillage, et une plus grosse capacité de mouillage à superficie égale (Arnaud 2016, p. 144-145). Mais plus la profondeur du mouillage est faible moins l'amplitude de la houle a besoin d'atteindre une hauteur de crête importante pour provoquer le talonnage du bateau. De plus, si la faible profondeur permet d'augmenter le nombre de mouillages, la zone située face à l'embouchure du Rhône est une zone absorbant un fort trafic. En conséquence, le stationnement de multiples navires, dans un espace (ou «lagon») circonscrit par les lobes alluviaux émergents, s'adapterait mal à la remonte des bateaux par vent favorable ou encore à leur descente.

14. Le sens étymologique grec du mot ancre signifie justement «ancre-crochet». fonds sableux des Saintes-Maries-de-la-Mer et la puissance des courants, les ancres à trois yeux intégrant deux pattes en bois devaient avoir un pouvoir d'accroche très peu efficace. Dans tous les cas, ces ancres ne peuvent pas correspondre à des navires de mer, même de faible tonnage (moins de $10 \mathrm{t}$ ), et demeurent en outre non datables en dehors de tout contexte archéologique car elles ont perduré longtemps sur de petites embarcations (Lima 2002, p. 59; Pomey, Gianfrotta 1981, p. 297-309). Parmi les jas d'ancre en plomb, cinq de plus de 1,50 m d'envergure ont été découverts (voir en dernier Long, Duperron 2011a, p. 59). En raison de leurs dimensions importantes, il serait particulièrement intéressant de connaître l'emplacement précis de ces cinq ancres par rapport à l'embouchure du Rhône (Fig. 5) ${ }^{15}$. Sont-elles éloignées de l'embouchure ? Dans ce dernier cas, on pourrait projeter le scénario de Strabon et envisager que d'innombrables barques à rames délestaient les bateaux à l'ancre d'une partie de leur cargaison sans forcément s'exposer au courant du fleuve (Géographie, V, 3, 5). Ou sont-elles, au contraire, positionnées face à l'embouchure du Rhône? En dehors de la présence d'une zone de mouillage, d'autres explications peuvent être avancées pour situer leur présence. Face aux difficultés de navigation dans cette zone deltaïque, certaines manœuvres comme le touage pourraient en effet expliquer la perte d'ancres dans une zone située justement entre les bancs de sable et l'embouchure du Rhône ${ }^{16}$. En considérant également qu'il n'est pas rare de trouver des séries de sept ou huit ancres de ce type sur un même bateau (Pomey 1997, p. 87), on ne peut pas non plus négliger la possibilité que ces ancres isolées appartiennent à des bateaux encore recouverts par le sable.

Au total, avec un inventaire réduit à seulement cinq ancres assurées, l'interprétation d'une zone de mouillage ne paraît pas être très significative et d'autres hypothèses sont dès lors possibles pour expliquer leur présence. En dehors des ancres, quelles sont les autres possibilités pour appréhender une zone de mouillage?

\section{Absence des conserves de bord des marins}

L'étude du dépotoir portuaire recouvrant l'épave fluviale ArlesRhône 3 a mis en évidence des céramiques communes à pâte claire qui sont spécifiques aux marins. Le caractère exogène de ces «conserves de bord», consommées par les marins et rejetées pardessus bord, renvoie aux différentes dotations de bord des bateaux dont les ports d'attache sont à rechercher dans l'ensemble du bassin méditerranéen (Djaoui à par. 1, Djaoui, Capelli à par.). Le stationnement prolongé des bateaux entraîne ainsi le rejet d'un vaste répertoire de céramiques communes extrêmement rares, voire inédites ou encore très peu diffusées, ce qui facilite l'identification fonctionnelle. Parmi cette catégorie, les pots du Latium ou de Bétique correspondent à ce type de conserves que l'on retrouve sur différents sites portuaires tels que Narbonne, Arles, Fos, Marseille,

15. Il faut souligner que pour l'Antiquité il est impossible d'associer les dimensions d'une ancre à une taille de bateau.

16. On retiendra en particulier les péripéties du capitaine Pierre Giot, dont les manuscrits datant de la fin du XVIII ${ }^{\mathrm{e}} \mathrm{s}$. - début du $\mathrm{XIX}^{\mathrm{e}} \mathrm{s}$. décrivent la procédure du touage pour se dégager d'un banc de sable (Payn-Echalier, Rigaud 2016, p. 44-46). Le bateau, engravé sur un banc, déploie une chaloupe en charge de porter une ancre le plus loin possible pour que le bateau puisse tirer dessus et s'extraire de cette fâcheuse posture. Bénéficiant d'une houle et d'un vent forcément portant, car le bateau s'engageait dans la remontée du Rhône, cette chaloupe ne pouvait guère que se diriger à proximité de l'embouchure, c'est-à-dire dans la zone où on localise les ancres antiques. Chaloupe qui naufrage avec l'ancre, aussière qui lâche sous la traction du bateau constituent autant d'explications pour situer ces ancres isolées à l'emplacement actuel de leur découverte. 

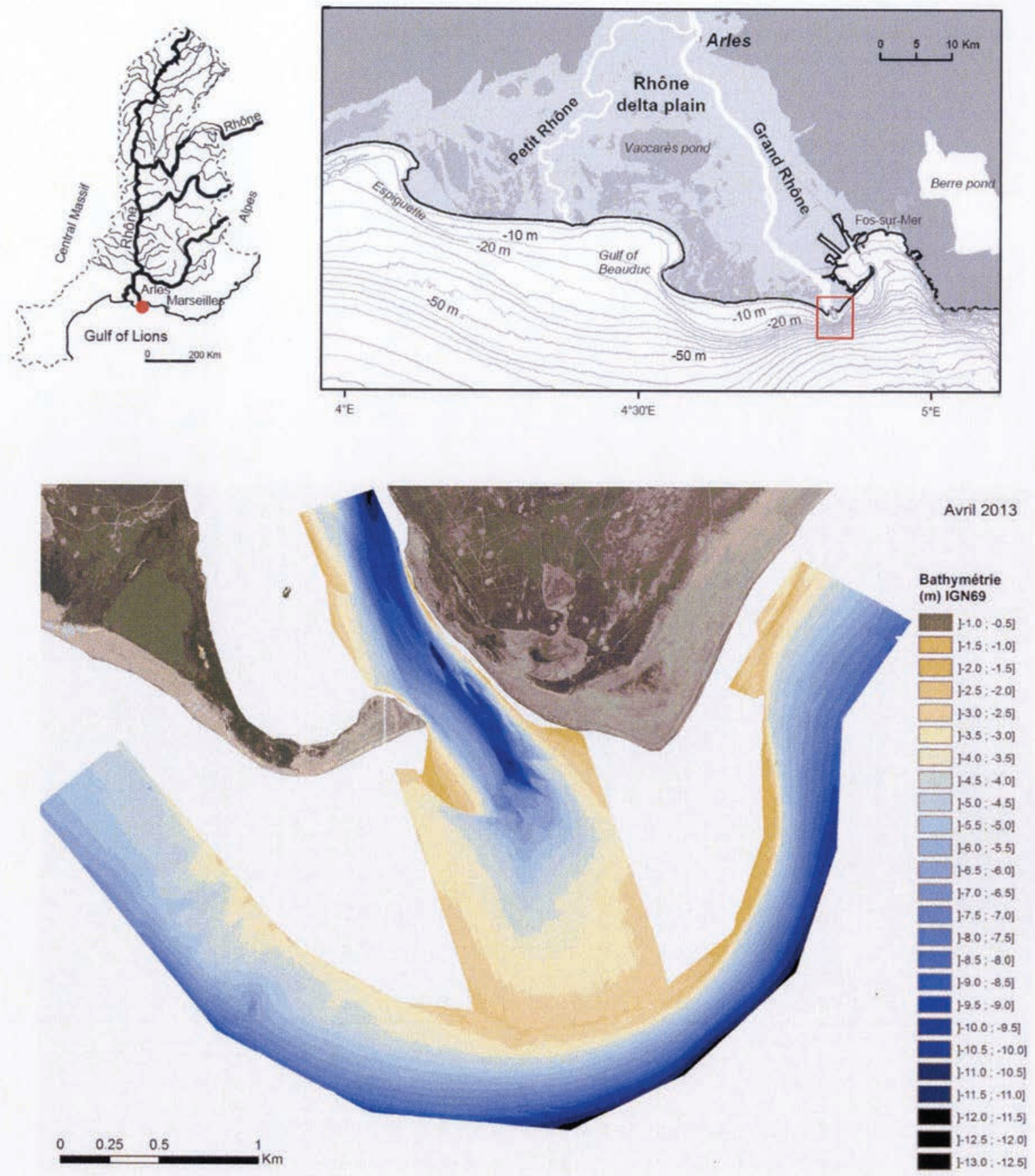

Fig. 6: Localisation et morphologie de l'embouchure du Grand Rhône (campagne de mars 2012) (Sabatier, Kulling à paraître).

Fréjus et Ostie, ou encore sur des épaves de Méditerranée nord-occidentale (Djaoui et al. 2014, Djaoui 2016). Concernant les différents sondages pratiqués dans la dite «zone de mouillage» des Saintes-Maries-de-la-Mer, les céramiques communes du HautEmpire sont pratiquement absentes à l'inverse des céramiques culinaires et fines qui regroupent des formes très diffusées telles que les marmites italiques à lèvre pendante, ou encore les culinaires africaines (14 exemplaires de la marmite Hayes 196) (Long, Duperron 2011b). Autrement dit, l'absence des pots du Latium et de Bétique s'accorderait mal aux stationnements prolongés des bateaux en provenance des ports de Rome ou d'Hispalis.

\section{ANALYSE DES ÉLÉMENTS FACTUELS QUI DÉFINISSENT UNE ZONE DE TRANSBORDEMENT}

La présence d'amphores face à l'entrée de Port-Gardian, au niveau du port des Saintes-Maries-de-la-Mer (Fig. 3, localisation SM0), est interprétée comme une zone de transbordement des marchandises (Long Duperron 2011b). Néanmoins, une observation de l'ensemble de ce dépotoir amphorique, permet d'envisager une autre hypothèse. 


\section{Quantité et chronologie des amphores}

Si les différentes missions menées en 2006, 2009 et 2010 ont permis de recenser 276 amphores fragmentées devant les SaintesMaries-de-la-Mer (gisement «SM0»), les quantités, la densité ou encore l'homogénéité de ces contenants, dont les datations s'étendent sur 1200 ans (du VI ${ }^{\mathrm{e}}$ s. av. J.-C. au VI ${ }^{\mathrm{e}}$ s. ap. J.-C.) (Ibid., p. 97-118), atténuent considérablement la portée des conclusions. La présence désordonnée et anachronique de ces amphores sur une aussi longue période pourrait révéler davantage un flux commercial ininterrompu qu'une zone de rupture de charge impliquant des quais et des zones de stockage dans un espace deltaïque aussi instable. D'autres explications sont possibles. Il semblerait logique, par exemple, qu'avant d'entamer la remontée jusqu'à Arles, les bateaux s'allègent aussi bien des amphores cassées pendant le transport que de celles consommées par les marins. De plus, en cas d'engravement sur un cordon alluvionnaire, il devait être impératif d'alléger rapidement le bateau en jetant les produits pondéreux les plus accessibles. La lex Rhodia de iactu prévoyait justement, pour les bateaux en situation de détresse, de jeter des marchandises par-dessus bord (Rougé 1966, p. 397-413; Ehmig 2014). Pour être efficace le jet doit être conséquent et ne pas se limiter à deux ou trois amphores mais concerner tout ou partie d'une cargaison, ce qui donne sous l'eau des amas d'amphores ou une distribution en chapelet ${ }^{17}$. La présence majoritaire d'amphores des $\mathrm{I}^{\mathrm{er}}$ et II ${ }^{\mathrm{e}}$ s. face à l'embouchure du Rhône SaintFerréol s'expliquerait davantage par un trafic important pour cette période que par la présence d'une zone de transbordement des produits amphoriques. On retiendra enfin que cette zone de rupture de charge suppose le stationnement de bateaux qui n'étaient pas en mesure de franchir les barres du Rhône. Qu'en est-il de l'architecture des bateaux échoués sur les barres d'avant côte?

\section{L'architecture des bateaux}

$\mathrm{Si}$ on rejette l'hypothèse d'une zone de transbordement, cela implique que les différents bateaux qui se sont échoués sur les cordons alluvionnaires cherchaient à pénétrer dans le delta du Rhône et devaient, par conséquent, présenter les caractéristiques architecturales pour passer directement les barres du Rhône. C'est précisément ce que semble indiquer une étude en cours réalisée par Sabrina Marlier sur les épaves chargées de fer présentant des vestiges de coque conservés (Marlier à par.). Ainsi, l'épave SM24, pour laquelle un fond de carène de 10 x $5 \mathrm{~m}$ a été conservé, correspondrait à un navire de type fluviomaritime d'une longueur estimée entre 20 et $25 \mathrm{~m}$ et caractérisé par une quille plate et un fond plat. C'est aussi dans le même ordre de grandeur qu'il faudrait inscrire $S M 2$, qui présente des échantillonnages de bois et des caractéristiques architecturales similaires à ceux de SM24. Ces navires seraient les plus gros navires à chargement de fer retrouvés en Camargue. Les dimensions modestes du bateau SM10, au tonnage estimé par Luc Long à 5 ou 6 tonnes, et de SM1, chargé de cent lingots de plomb, correspondraient en revanche à de petites unités. Dans tous les cas, il est vraisemblable que ces navires, dans des conditions normales (météo, vent favorable, barre suffisamment profonde, etc.), devaient pouvoir franchir la barre du Rhône.

Concernant les autres épaves, aucun vestige de coque n'a été retrouvé. Si c'est la forme de la carène qui définit mieux le tirant d'eau que le tonnage lui-même, certaines remarques peuvent être formulées sur la capacité des bateaux à franchir la barre du Rhône.

17. Information donnée par Patrice Pomey que je remercie.
Contrairement aux plus imposantes cargaisons de marbre, comme celle du navire échoué près de Saint-Tropez avec un poids estimé de 200 t (Benoit 1952, p. 240-244), les trois navires SM18, SM21, SM22 semblent, au contraire, être «calibrés» sur un tonnage moyen, estimé entre 30 et 40 tonnes et regroupant entre six et sept blocs maximum ${ }^{18}$. Ces embarcations lapidaires et leur chargement pouvaient très vraisemblablement avoir été constitués pour franchir les barres du Rhône ${ }^{19}$ dont on estime, selon les sources modernes, le niveau à 2,60 $\mathrm{m}$ de profondeur ${ }^{20}$. Dans tous les cas, il est impossible de considérer que ces bateaux essayaient de gagner la zone de mouillage située face à l'embouchure du Rhône Saint-Ferréol (cf. supra), pour transborder des blocs de marbre de plusieurs tonnes sur des allèges. À l'évidence, ces bateaux devaient bien franchir les barres du Rhône.

\section{ANALYSE DES ÉLÉMENTS FACTUELS QUI DÉFINISSENT LES STRUCTURES PORTUAIRES DES SAINTES-MARIES-DE-LA-MER}

Dès 2006, la découverte de blocs en calcaire, dont certains étaient dotés d'un orifice central (Long 2008, p. 44), avait conduit Luc Long à les interpréter comme la base d'édifices en bois comparables à ceux restitués dans l'anse Saint-Gervais à Fos (Gassend, Maillet 2004). Mais contrairement à ces derniers, les différents blocs recensés aux Saintes-Maries-de-la-Mer ne sont pas scellés dans une maçonnerie. Autrement dit ces pierres, trouvées directement au contact du sable, ne sont pas en place et ne présentent aucune orientation précise ${ }^{21}$. L'identification de ces blocs en tant que structures portuaires demeure par conséquent hypothétique. De la même façon, les «fragments de tegulae » trouvés à proximité de ces blocs ne semblent quantitativement pas assez significatifs pour y être associés. De plus, la dénomination «clous de charpente» pour les clous trouvés dans le même secteur constitue déjà un parti-pris. Il est en effet impossible d'attribuer des clous isolés à des clous de charpente ou à des clous de bateaux. Outre l'instabilité sédimentaire de cette zone deltaïque, qui ne se prête guère à l'édification de structures pérennes ${ }^{22}$, l'idée de délester les pondéreux des bateaux maritimes sur des allèges qui déchargent à nouveau les matériaux dans des hangars situés à proximité immédiate ne semble ni fonctionnel, ni logique. Et pourquoi des bateaux maritimes prendraient-ils le risque de passer entre les cordons alluvionnaires pour décharger les marchandises dans ces hangars alors qu'ils pouvaient rester au large pour les transborder sur des allèges?

18. Pour $S M 28$, il est impossible de savoir si cette épave transportait un seul bloc de marbre de 30 ou 40 tonnes (Long, Duperron 2016, p. 203, note 5).

19. Pour faciliter la pénétration dans l'embouchure du Rhône et la remontée du fleuve jusqu'à Lyon, il est intéressant de souligner que les navires à dolia (10 av. J.-C./40 apr. J.-C.), avaient un port en lourd calibré entre 50 et 63-66 tonnes et des dimensions réduites (18-22 $\mathrm{m}$ de longueur pour 6-7 $\mathrm{m}$ de large).

20. Voir le chapitre «le problème du tirant d'eau pour le passage de la barre du Rhône » dans Marlier 2008, p. 167-168.

21. Luc Long explique bien la difficulté d'interpréter ces blocs au sein d'un milieu très perturbé : «Le passage répété des chalutiers devant Port-Gardian, dans les années 1980, a vraisemblablement effacé ou mutilé un grand nombre de ces alignements. En outre, les roches naturelles étant absentes de Camargue, sur l'ensemble de son immense territoire, on peut imaginer qu'une partie des pierres calcaires a été récupérée depuis l'Antiquité pour fabriquer de la chaux, des maisons, ou stabiliser les chaussées » (Long 2008, p. 42).

22. La ligne de rivage n'a jamais été établie précisément en liaison avec ces structures. Seul le positionnement des épaves antiques permet de situer la côte à une distance comprise entre 100 et $200 \mathrm{~m}$ (Long, Illouze 2004, p. 322). 
Concernant la présence de ces blocs, plus ou moins éparpillés, plusieurs explications sont possibles, comme la pratique du jet avec un effet de dispersion en chapelets ( $c f$. supra), la signalisation ou le balisage des chenaux navigables ${ }^{23}$, ou encore la présence d'une cargaison lithique d'une épave disloquée par un chalut.

À partir de ces données contradictoires, une prise de recul semble nécessaire pour projeter non plus un regard focalisé sur le seul Rhône Saint-Ferréol, mais plutôt un regard panoramique sur l'ensemble du delta du Rhône et déterminer ainsi les autres possibilités pour acheminer, transférer et stocker les différents produits.

\section{LES AUTRES PORTS POTENTIELS DU DELTA DU RHÔNE}

Par le mot «port» il est nécessaire de reprendre la définition qu'en donne Pascal Arnaud en les décrivant «comme une simple interface entre la terre et la mer en vue de l'activité maritime des hommes » (Arnaud 2016, p. 140). Autrement dit, le port n'implique pas forcément «la présence d'infrastructures portuaires de grand style», ou des quais en grand appareil, mais peut se limiter, dans l'exemple d'une embouchure, au transbordement d'une cargaison d'un bateau à un autre (Arnaud 2016, p. 139-140).

\section{LES PORTS NON PROTÉGÉS ET/OU LES ZONES D'ATTENTE DES EMBOUCHURES}

Après avoir rejeté l'hypothèse d'un port abrité par des lobes émergents situé face à l'embouchure du Rhône Saint-Ferréol, je propose une autre hypothèse: celle d'un port de type salos, c'est-àdire un plan d'eau situé plus au large de l'embouchure et dès lors davantage ouvert à la houle (Arnaud 2016, p. 140-141). Cette exposition à la houle suppose que ce type de port devait plutôt se constituer par mer calme à faiblement agitée.

\section{Au large du Rhône Saint-Ferréol}

En reprenant la carte bathymétrique de l'embouchure du Grand Rhône actuel, on constate qu'aux profondeurs de -8/10 m NGF les bateaux peuvent s'éloigner de l'emprise des courants et des cordons alluvionnaire du delta tout en maintenant une profondeur suffisante et stable pour assurer un ancrage de qualité. Dans le système hiérarchique des ports fluviomaritimes définis par Pascal Arnaud, il s'agit d'un port de type salos. Cette configuration était celle d'Ostie qui ne disposait pas d'un plan d'eau calme de type $\operatorname{limèn}^{24}$, mais d'un port situé plus au large. Strabon $(\mathrm{V}, 3,5)$ précise qu'à

23. Un texte de Rutilius Namatianus (Red. 457-462), cité par Pascal Arnaud (2016, p. 146), illustre un mode simple et souple de balisage des passes navigables entre les bancs de sables à Vada Volterrana (Vado Ligure) dans son voyage de l'automne 417. On y apprend notamment que des hauts lauriers étaient fichés (dans le sable? dans des blocs troués ?) de façon à offrir un visuel dans cet amas de récifs de limon. Autrement dit, les pierres trouées des Saintes-Maries-de-la-Mer, dont certaines seraient cimentées, pourraient avoir servi de support à des pieux ou des arbres pour signaler les chenaux navigables.

24. Les textes antiques présentent en effet l'embouchure du Tibre comme «alimenos», c'est-à-dire sans port de type limen. Dion Cassius (LX, 11) indique ainsi que la région des bouches du Tibre n'offrait ni plages de débarquement sûres, ni ports convenables. De la même façon, Denys d'Halicarnasse affirme que le Tibre se jette sur les rivages sans ports et exposés que forme la mer Tyrrhénienne (Ant. Rom. III, 1). cause des dépôts d'alluvions sans cesse croissants «les navires restent par conséquent au large et y mouillent en grande rade [...], les innombrables barques à rame qui déchargent les cargaisons et en apportent d'autres en échange permettent aux navires de repartir rapidement, sans avoir à s'exposer au courant. Certains d'entre eux, aussi, s'allègent d'une partie de leur chargement et pénètrent ensuite dans l'embouchure du Tibre pour remonter jusqu'à Rome...». Avant la construction du port artificiel de l'empereur Claude, situé à $3 \mathrm{~km}$ au nord de l'embouchure du Tibre, cette opération de transbordement était jugée en effet par Strabon comme très périlleuse et seul l'appât du profit pouvait avoir raison des dangers. Il est vrai qu'en plus des contraintes deltaïques de l'embouchure du Tibre, la côte du Latium présente également un tombant rapide qui réduit considérablement l'espace du mouillage et rendait ainsi les manœuvres de transbordement particulièrement dangereuses en cas de coups de vent (Boetto 2006, p. 396). Ce dernier point est particulièrement intéressant car, à l'inverse du Latium, le littoral de Camargue s'étend sur plusieurs kilomètres en maintenant un pendage faible. Sur plus de $2 \mathrm{~km}$ de la côte les profondeurs sont en effet inférieures à $15 \mathrm{~m}$, ce qui devait favoriser un mouillage en grande rade. Autrement dit, la possibilité de mouiller au large des Saintes-Maries-de-la-Mer permettait aux bateaux les moins aptes à franchir les barres du Rhône non seulement de s'affranchir des difficultés du delta mais également de disposer d'un large espace où s'ancrer.

\section{Au large des deux autres embouchures}

De la même façon que pour le Rhône Saint-Ferréol, les embouchures de Daladel et d'Ulmet devaient présenter les conditions géographiques similaires pour permettre aux bateaux de s'éloigner du delta en s'ancrant au large. Par mer calme à modérément agitée, les bateaux maritimes dans l'incapacité de franchir les barres du Rhône devaient privilégier ces ports en eau profonde pour transborder les marchandises, tandis que par mer agitée et/ou tempête, le golfe de Fos et l'anse Saint-Gervais offraient une zone protégée plus apte à ce type de manœuvres.

\section{LES PORTS PROTÉGÉS ET/OU LES ZONES D'ATTENTE}

\section{Le golfe de Fos}

Le golfe de Fos offre, à l'est du delta, une baie qui demeure assez bien protégée des deux vents dominants: le vent de sud-est et le vent du nord-ouest (Mistral). Certaines hypothèses envisagent d'ailleurs que l'actuel étang du nord-ouest de l'Estomac aurait pu être utilisé comme un port naturel bien protégé en fond de baie (Vella et al. 2016). Comme le signale Jacques Rossiaud pour le Moyen Âge, «les relations d'Arles avec la mer sont déterminées par la configuration des graus et l'on a retenu à ce propos que les Arlésiens avaient depuis longtemps choisi la régularité au détriment des portées parce qu'ils disposaient non loin de Passon d'un avant-port réputé sûr: Bouc [...]. Bouc a été très longtemps considéré, après l'ensablement d'Aigues-Mortes, comme le seul refuge accessible aux vaisseaux entre la Catalogne et Marseille » (Rossiaud 2007, p. 181).

D'un point de vue archéologique, il est en revanche encore extrêmement difficile de se faire une idée précise tant de la chronologie

Pour la définition du port de type limen, voir Rougé 1966; Arnaud 2010, p. 108; Arnaud 2016, p. 140. 


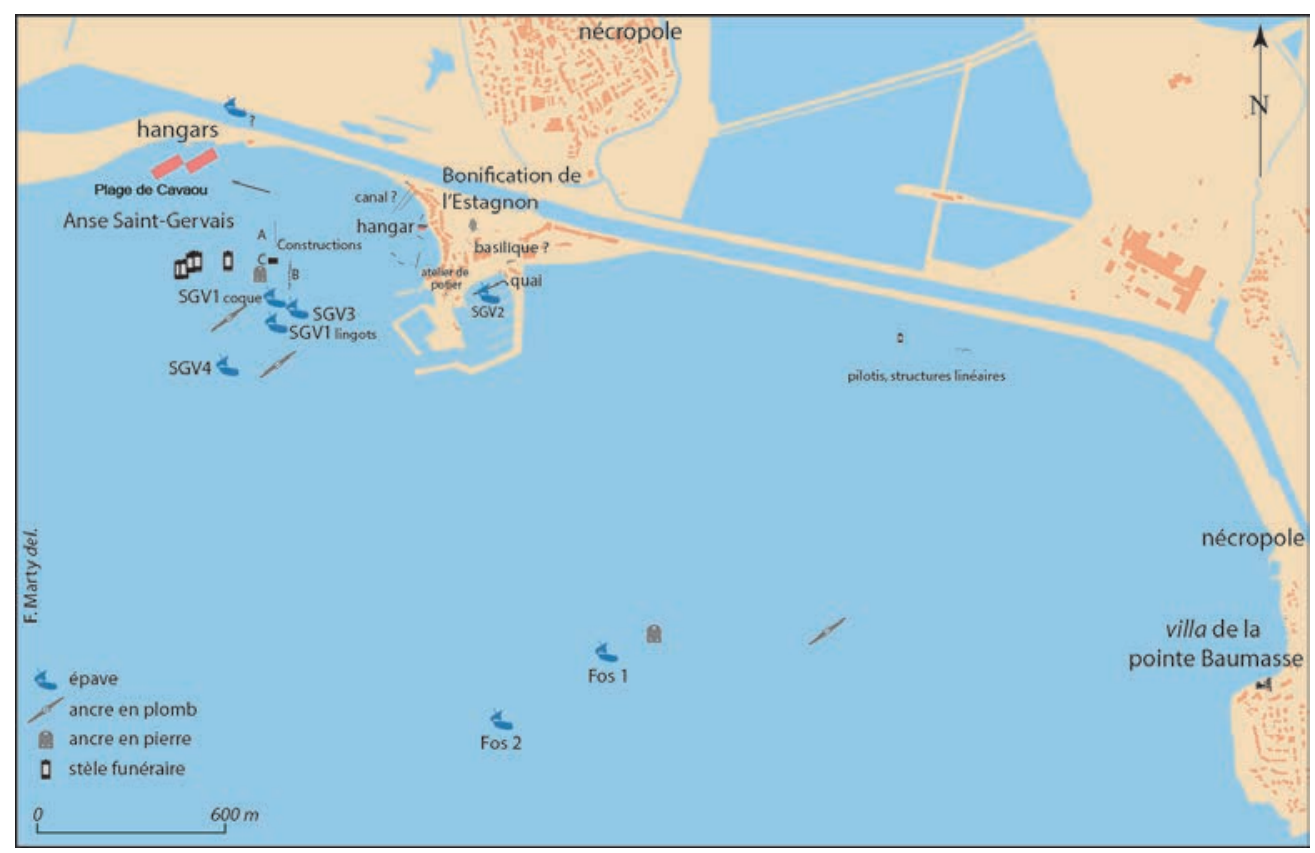

Fig. 7: Vestiges antiques du littoral de Fos-sur-Mer (DAO F. Marty / PIPC Istres Ouest Provence).

que de l'identification des structures, et donc de l'organisation de cet immense complexe portuaire ${ }^{25}$. Si l'on suit les sources anciennes (Tréziny 2004, p. 98-102), la fondation du port de Fos serait liée à la création du canal de Marius en 102 av. J.-C. (Plutarque, Vie de Marius, 15, 2-4; Strabon, IV, 1, 8). Canal dont il est difficile d'évaluer la date d'achèvement ${ }^{26}$ mais qui, dans tous les cas, fonctionnait encore au moment de sa cession aux Marseillais par César, en 49 av. J.-C. (cf. infra). L'étude du matériel recueilli dans l'anse SaintGervais depuis la fin des années 1940, et notamment de la sigillée italique (Legendre 2015), suggère toutefois que le démarrage effectif de l'activité portuaire n'est pas antérieur à 20 av. J.-C. (F. Marty, communication personnelle; Marty, Zaaraoui 2009, p. 422-423). On recense néanmoins deux épaves plus anciennes, Fos 1 et Fos 2 (datées vers 50-30 av. J.-C.), aux côtés de trois épaves du Haut-Empire, Saint-Gervais 4 ( ${ }^{\mathrm{er}}$ s. ap. J.-C.), Saint-Gervais 3 (milieu du $\mathrm{II}^{\mathrm{e}}$ s. ap. J.-C.) et Saint-Gervais 1 (138-139 ap. J.-C.) (Giacobbi-Lequément 1997; El-Amouri et al. 2015, p. 134; Gateau 1996, p. 187), et une épave plus tardive du VII siècle, Saint-Gervais 2 (Jézégou 1998) (Fig. 7).

Il est intéressant de souligner que les fouilles sous-marines restituent dans le golfe de Fos (pointe Saint-Gervais) un faciès céramique identique à celui du Rhône, avec notamment un grand nombre de céramiques que l'on attribue à la dotation de bord des marins en provenance de Bétique (Djaoui et al. 2014 ; Djaoui à par. 2, Djaoui, Capelli à par.). Ces « conserves de bord $»^{27}$ en provenance d'Hispalis matérialisent ainsi soit des navires fluviomaritimes qui attendaient les vents favorables dans le golfe de Fos avant d'atteindre Arles, soit des bateaux maritimes qui n'avaient pas la

25. Gateau 1996, p. 184-185; El Amouri et al. 2015; Fontaine et al. à par.

26. Il est peu probable que cette œuvre monumentale ait été achevée en seulement trois ans. Frédéric Marty souligne en effet que les travaux de construction du canal de navigation d'Arles à Bouc, réalisés avec l'aide de condamnés aux travaux publics, ont commencé en 1803 pour une mise en service en 1834, après une interruption entre 1813 et 1822 (Marty, Zaaraoui 2009, p. 422).

27. $C f$. p. 133 dans cet article. capacité de franchir les barres du Rhône et qui devaient s'ancrer pour décharger les produits amphoriques à Fos.

\section{L'anse des Laurons}

Située à l'est du golfe de Fos, l'anse des Laurons (commune de Martigues) est formée de trois criques rocheuses dominées par trois collines qui permettaient aux navigateurs de s'abriter des vents d'est et du sud (Ximénès, Moerman 1988). Avec une superficie de $100000 \mathrm{~m}^{2}$, l'étendue du bassin se rapproche de celui de Forum Iulii (Fréjus) et Cemtumcellae (Civitavecchia) ${ }^{28}$. Si des bâtiments situés dans la crique nord ont été attribués à une villa maritime, il est en revanche «difficile d'établir des liens réels fonctionnels et chronologiques avec le port ainsi qu'avec un bâtiment de stockage de $500 \mathrm{~m}^{2}$ situé au bord du rivage antique de la crique sud» (Moerman 1993, p. 19-23). Mais quel que soit le statut de ce port, privé ou pas, il devait participer au complexe portuaire de Fos et recevoir des bateaux en délestage du port principal.

Les quatre épaves datées de l'anse des Laurons s'inscrivent dans une chronologie plus tardive que celle de notre problématique (épaves Laurons 1, 2, 3 et 4) ${ }^{29}$. Leur espace de navigation englobe la remontée du Rhône (Marlier à par.). Ces embarcations, en attente probable de chargement d'une cargaison (lithique?), pouvaient attendre les vents favorables et emprunter l'un des bras du Rhône. Dans cette zone protégée, une situation analogue est tout à fait envisageable pour le $\mathrm{I}^{\mathrm{er}}$ s. ap. J.-C. Bien qu'une étude quantitative du

28. Dans l'Itinéraire Maritime d'Antonin, ce port pourrait correspondre à Dilis Positio (Ximénès, Moerman 1988, p. 128) ou encore à celle de Carro (Arnaud 2010 p. 109).

29. Les épaves de l'anse des Laurons sont datées du $\mathrm{III}^{\mathrm{e}} / \mathrm{IV}^{\mathrm{e}}$ s. ap. J.-C. pou Laurons 1; de la fin du $\mathrm{II}^{\mathrm{e}}$ s. ap. J.-C pour Laurons 2; du $\mathrm{IV}^{\mathrm{e}}$ s. ap. J.-C. pour Laurons 3, du IV ${ }^{\mathrm{e}}$ s. ap. J.-C pour Laurons 4 (Gassend et al.1984 ; Ximénès, Moerman 1987; Pomey (dir.) 1987-1988, p. 12-13 et pour les structures portuaires p. 21-24; Pomey (dir.) 1992, p. 24-25). Les autres épaves (Laurons 5, 6, 9 et 10) n'ont conservé en revanche aucun vestige de coque apparent ou d'éléments de datation. 
nombre d'amphores fasse encore défaut, il semblerait que le dépotoir portuaire de l'anse des Laurons témoigne d'une fonction commerciale importante ${ }^{30}$.

\section{LA PRÉSENCE DE PORTS SPÉCIALISÉS?}

Différencier les embouchures en fonction de la nature des cargaisons devait permettre d'étendre et de spécialiser les espaces portuaires dans le but d'emmagasiner le plus possible les marchandises avant la période du mare clausum (Djaoui à par. 3). Cette différenciation devait impliquer non seulement l'existence de ports spécialisés, avec des quais et des engins de levage adaptés, notamment pour les pondéreux, mais également des zones de contrôle, de stockage et de transformation (Djaoui à par. 3).

\section{Des ports spécialisés au Rhône Saint-Ferréol?}

Face aux millions d'amphores qui transitaient par Arles, il a probablement été opportun d'éloigner les matériaux encombrants (marbre et bois) de l'espace portuaire de l'antique Arelate $^{31}$. Si au $\mathrm{I}^{\text {er }}$ siècle apr. J.-C. le port était très vraisemblablement saturé par le trafic alimentaire ( $c f$. infra), le stockage et la transformation des pondéreux ont dû être repoussés, soit en périphérie ${ }^{32}$, soit beaucoup plus en aval, c'est-à-dire au niveau de la section inférieure du Rhône Saint-Ferréol (Djaoui à par. 3). Pour les métaux, que ce soit par la convergence des multiples bateaux assurant leur transport ou par leur «croisement» sur le même bras, la section inférieure du Rhône Saint-Ferréol apparaît également comme un espace idéal pour recevoir, contrôler (portorium?) et redistribuer ces marchandises, aussi bien en direction d'Arles que vers Rome (Djaoui à par. 3).

L'hypothèse d'un port situé dans la section inférieure du Rhône Saint-Ferréol correspondrait au troisième type de ports fluviomaritimes défini par Pascal Arnaud. Aux ports qui sont situés devant l'embouchure (type salos ou limen) et à ceux qui supposent une remontée à proprement parler, c'est-à-dire, ici, jusqu'à Arles, viennent en effet s'ajouter les ports pour lesquels l'embouchure constitue un chenal d'accès inférieur à $4 \mathrm{~km}$ à l'intérieur des terres. (Arnaud 2016, p. 142). Il faut souligner que la présence d'un tel port ne devait nullement empêcher les allèges, portées par un vent favorable, de remonter directement le Rhône jusqu'à Arles (Djaoui à par. 3).

D'un point de vue pratique, les bateaux pouvaient profiter d'un méandre du Rhône pour décharger les matières premières et repartir. Dans la partie convexe, les bateaux bénéficiaient en effet d'un courant faible mais d'une profondeur limitée et dans la partie concave d'une profondeur importante mais d'un courant plus fort.

\section{Fos: Portus uinarius et olearius?}

Sur la Table de Peutinger le port de Fos est représenté de la même façon que le port d'Ostie: un bâtiment à quatre nefs qui évoque très certainement des horrea (Tchernia, Viviers 2000, p. 781). Tout

30. Ximénès et Moerman 1988, p. 128 notent en effet la présence d'amphores issues de l'ensemble du bassin méditerranéen, Gaule, Espagne, Italie, Afrique du nord, îles grecques et Alexandrie, dans une chronologie comprise entre le milieu du $\mathrm{III}^{\mathrm{e}}$ av. J.-C. et le $\mathrm{VI}^{\mathrm{e}} \mathrm{s}$. ap. J.-C., avec une prédominance toutefois pour le Bas-Empire.

31. Pour un complément, voir Djaoui à par. 1.

32. Eloigner les pondéreux des centres urbains, au profit des comestibles, était encore l'une des préoccupations du Consulat de 1738 à Lyon (Conchon 2007, p. 51-52). comme l'alimentation de Rome l'exigeait, l'axe Rhône-Saône-Rhin devait posséder des zones tampons de stockage pour absorber une demande massive. Si pour Rome les mentions portus uinarius $(C I L$ VI, 9189-9190-37807) et portus olearius (AE 1980, 84) ${ }^{33}$ indiquent des zones de déchargement spécialisées dans le vin et l'huile, les découvertes du golfe de Fos suggèrent également une telle convergence. La réutilisation massive des amphores à l'Estagnon (anse Saint Gervais à Fos) pour la bonification d'un terrain, ou encore la redistribution des produits amphoriques dans des cruches et/ou des pots, témoignent d'un tel stockage (Djaoui à par. 3 ; Djaoui, Capelli à par.).

Concernant les aménagements portuaires, les fouilles menées par Jean-Marie Gassend dans l'anse Saint-Gervais, dans la zone ouest du golfe de Fos, ont permis de localiser sur la plage de Cavaou deux structures quadrangulaires de $110 \mathrm{~m}$ sur $36 \mathrm{~m}$, interprétées dans un premier temps comme des naualia ${ }^{34}$. Cette identification est contestée ${ }^{35}$ et on a suggéré d'y voir plutôt des hangars ${ }^{36}$. Depuis 2012, l'anse Saint-Gervais et le site de la Marronède (à l'est du golfe de Fos) font l'objet de nouvelles recherches ${ }^{37}$. Au centre de l'anse, plusieurs ensembles architecturaux de grandes dimensions ont été ainsi mis en évidence. Si l'interprétation de ces structures doit encore être précisée, la reprise globale du dossier laisse beaucoup d'espoir pour la compréhension de cette immense zone portuaire.

\section{LE PORT SITUÉ À LA TÊTE DU DELTA: ARELATE}

Les différentes missions d'expertise et de carte archéologique dirigées par Luc Long depuis 1985 ont permis de localiser dans le Rhône quatorze épaves (Long, Duperron 2013). La présence d'une brêle de radeau antique (Arles Rhône 10), de deux chalands (AR3, et $A R 5)$, de trois navires fluviomaritimes ( $A R 7, A R 8$ et $A R 14)$ et d'un bateau de mer (AR13) restitue bien la variété des types de bateaux correspondant à cet espace de navigation ${ }^{38}$. Au corpus des fluviomaritimes et des petits bateaux de mer s'ajoutent également des découvertes matérielles qui attestent un flux commercial direct en provenance de la Méditerranée.

33. L'emplacement du portus uinarius n'est pas connu mais il est toutefois admis qu'il devait se situer proche du Tibre et en aval du centre de Rome. Un deuxième portus uinarius superior (CIL XI, 3156) pourrait être situé en amont du centre de Rome (Andreau, 1987, p. 166) pour recevoir les importations en provenance de la péninsule italienne par voie fluviale.

34. Il s'agit d'une importante construction en gros blocs régulièrement taillés alignés mais disjoints (Gateau 1996, p. 184-188; Gassend, Maillet 2004).

35. La ligne de rivage n'a jamais été établie précisément en liaison avec ces structures, et le plan des bâtiments ne correspond pas à celui de naualia. Dans ces derniers, les bateaux ne sont jamais placés les uns derrière les autres (Pomey 1992, p. 18).

36. Un troisième hangar de dimensions plus réduites est également signalé (Sciallano, Leffy 1992, p. 42).

37. Ces investigations, conduites sous la direction de Souen Fontaine (Drassm), Mourad El Amouri (Ipso Facto) et Frédéric Marty (PIPC Ouest Provence), s'intègrent dans le PCR «Fossae Marianae: le système portuaire du golfe de Fos et le canal de Marius » (El Amouri, Fontaine, Marty 2015; Fontaine et al. à par.).

38. Il faut noter que le site $A R I$ ne correspond pas à une épave mais à un dépotoir antique lié à un aménagement de berge (Long, Duperron 2013). La documentation n'a pas permis de déterminer le type architectural des navires des autres épaves expertisées (AR2, AR4, AR6, AR9, AR11, AR12 et AR15) (Long, Duperon 2013; Marlier à par.). 


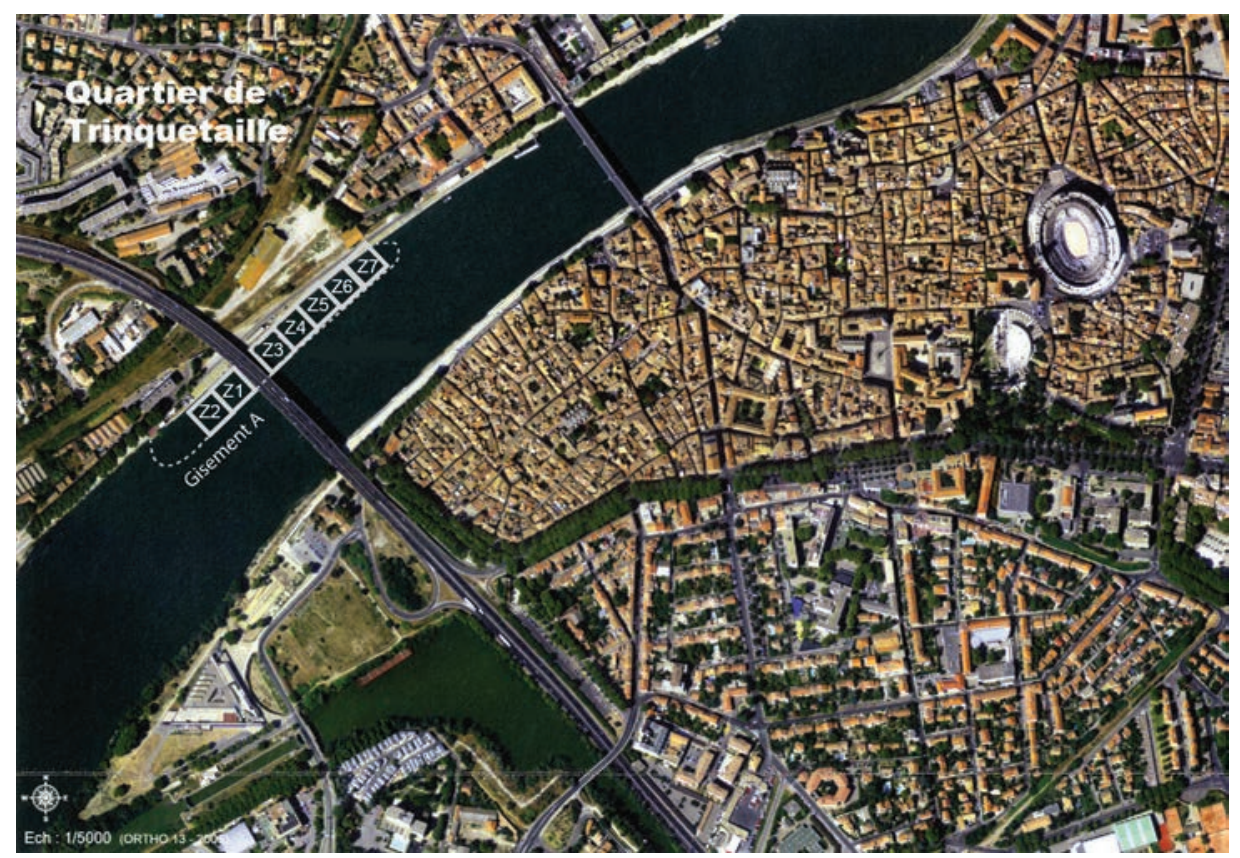

Fig. 8: Vue aérienne de la ville d'Arles avec les délimitations du dépotoir portuaire de Trinquetaille (gisement A), situé en rive droite du Rhône, et les différentes zones de fouille dirigées par L. Long. (๑ Parc naturel régional de Camargue et Dynamiques Écologiques et sociales en milieux deltaïque, D.A.O. : David Djaoui).

\section{La preuve matérielle d'une navigation entre la Méditerranée et Arles}

La découverte dans le Rhône des «conserves de bord» en provenance de Bétique et du Latium confirme que des bateaux de type fluviomaritime, ou peut-être également des petites embarcations maritimes, partaient d'Hispalis et des ports de Rome, et remontaient directement le Rhône jusqu'à Arles (Djaoui 2016; Djaoui Capelli à par.). Ces récipients en céramique commune claire, absents des contextes domestiques arlésiens, et dont on peut supposer qu'ils étaient embarqués par les marins juste avant de partir, identifient le port exportateur. Sur l'un des pots de Bétique, le graffito DIVRNI, la ration quotidienne, exprime bien leur fonction alimentaire.

\section{L'image d'un port flavien «saturé»}

La cité fluviale d'Arles recevait, durant le Haut-Empire, des millions d'amphores provenant de l'ensemble du bassin méditerranéen. Ce trafic immense a généré un vaste dépotoir amphorique, baptisé Gisement A, qui s'étend de part et d'autres du pont de la voie rapide $(\mathrm{N} 113)^{39}$. À l'inverse de la rive gauche, inaccessible aux fouilles en raison d'une accumulation d'énormes blocs modernes renforçant la digue actuelle, la rive droite, jouxtant le quartier actuel de Trinquetaille, a fait l'objet de différentes campagnes de fouilles. Le dépotoir le plus ancien est celui qui est le plus éloigné du centre urbain (Zone 2). Plus on se rapproche de la ville, plus la chronologie des couches de surface livre un matériel récent. On passe ainsi de la zone 2, datée de la seconde moitié du I ${ }^{\mathrm{er}}$ s. apr. J.-C., à la zone 3, datée de 70 à 140 apr. J.-C., aux zones 5 et 6, datées du début du $\mathrm{V}^{\mathrm{e}}$ siècle apr. J.-C. (Long 2008, p. 117) (Fig. 8). L'homogénéité de la datation de la zone 2 et son éloignement du tissu urbain résulte très vraisemblablement de l'augmentation du volume commercial de la

39. Long, Piton, Djaoui 2009, voir en particulier la carte «Localisation des zones de fouille dans le gisement $\mathrm{A} »$ (Fig. 2, p. 571). période flavienne qui conduisait les bateaux à s'amarrer de plus en plus loin du centre-ville ${ }^{40}$. Si la présence massive de ces amphores dans le Rhône reflète une activité commerciale importante, les seules structures portuaires découvertes à l'aplomb des fouilles subaquatiques datent des II-III ${ }^{\mathrm{e}}$ s. apr. J.-C.41

\section{SYNTHÈSE}

Une première synthèse peut être proposée en prenant en compte aussi bien les embarcations fluviomaritimes et les petits bateaux de mer, qui avaient la capacité de remonter directement le Rhône à la voile ${ }^{42}$, que les plus grosses unités maritimes pour lesquelles le transbordement de leurs cargaisons sur des allèges était obligatoire. À cette donnée architecturale s'ajoute évidemment la nature des cargaisons, avec des bateaux chargés uniquement de matières premières (principalement pierre et bois) ou d'amphores, mais aussi présentant des cargaisons mixtes.

40. Contrairement aux bassins portuaires dont l'espace est parfaitement circonscrit, les ports fluviaux peuvent se développer sur toute la longueur des deux rives. Si on peut envisager deux, voire trois bateaux à couple, l'extension latérale de l'amarrage a ses limites: la force du courant qui augmente au niveau du chenal et l'obligation de disposer d'un chenal suffisamment large pour le passage des bateaux, montants et avalants, constituent en effet des contraintes importantes. Il est par conséquent assez logique que le développement de l'activité commerciale éloigne les bateaux du centre urbain. Cette extension est toutefois limitée par les tournants où s'accumulent les sédiments et limitent ainsi les tirants d'eau des bateaux.

41. Si d'importantes similitudes formelles avec les «Grandi Horrea» d'Ostie ont pu être évoquées sur le sondage 1, il n'a pas été possible d'identifier les marchandises stockées (Mellinand, Sivan 2011, p. 125-127).

42. C'est ce que semble montrer une étude en cours conduite par S. Marlier $(\mathrm{MdAa} / \mathrm{CD} 13)$ à partir de la reprise des données de l'ensemble des épaves du delta du Rhône (Marlier à par.). 
Selon ce modèle, on considérera que si le canal de Marius était bien encore en fonctionnement au $\mathrm{I}^{\mathrm{er}} \mathrm{s}$. ap. J.-C., les bateaux maritimes pouvaient remonter directement jusqu'à Arles en l'empruntant ${ }^{43}$.

\section{LES BATEAUX CHARGÉS DE MATIÈRES PREMIÈRES}

En provenance de Narbonne et très probablement d'Hispalis pour les métaux ${ }^{44}$, ou de la côte tyrrhénienne (Luni?), et, d'une façon générale, de Méditerranée orientale pour les marbres, ces bateaux pouvaient être chargés uniquement de matières premières. En fonction de leur type architectural, deux cas sont à envisager:

\section{Bateaux aptes à franchir les barres du Rhône et à remonter le fleuve (bateaux de type fluviomaritime et/ ou maritime avec un faible tirant d'eau) (Fig. 9)}

Par vent favorable, les bateaux pouvaient directement remonter le Rhône Saint-Ferréol. Et par vent défavorable, ils devaient rejoindre une zone d'attente que l'on situe à Fos, ou dans l'anse des Laurons, ou encore stationner en grande rade pour attendre les vents favorables.

\section{Non aptes à franchir les barres du Rhône (Fig. 10)}

Ces bateaux devaient stationner à Fos, ou dans l'anse des Laurons ${ }^{45}$ et transborder dans des allèges les matières premières en direction du Rhône Saint-Ferréol. À l'instar du témoignage de Strabon pour Ostie, on peut également envisager que ces bateaux, ancrés en grande rade, étaient délestés par une multitude d'allèges (en rouge sur la Fig. 10).

\section{LES NAVIRES CHARGÉS D'AMPHORES}

\section{Aptes à franchir les barres du Rhône (Fig. 11)}

Par vent favorable, les bateaux pouvaient directement remonter le Rhône de Daladel ou d'Ulmet. Et par vent défavorable, ils pouvaient rejoindre une zone d'attente que l'on situerait à Fos, ou dans l'anse des Laurons, ou encore stationner en grande rade pour attendre les vents favorables.

\section{Non aptes à franchir les barres du Rhône (Fig. 12)}

Plusieurs cas peuvent se présenter. Les bateaux pouvaient transborder les amphores dans des allèges à Fos, ou dans l'anse des Laurons, et ensuite se dirigeaient en direction du Rhône d'Ulmet ou de Daladel (en noir sur la Fig. 12). Ce transbordement sur des allèges pouvait également s'effectuer au large de l'embouchure d'Ulmet ou de Daladel (en rouge sur la Fig. 12). Les bateaux pouvaient également décharger les amphores à Fos dans des zones «tampons» de stockage dont la durée dépendait, aussi bien des besoins alimentaires que des conditions météorologiques pour amorcer la remonte. Une dernière possibilité consistait à emprunter le canal de Marius, plus ou moins directement en fonction de

43. Avec l'exemple de Narbonne, on constate en effet que le chenal artificiel de $50 \mathrm{~m}$ de largeur présentait une profondeur de 3,50 $\mathrm{m}$ de profondeur au droit du quai et devait pouvoir accueillir des bateaux maritimes de fort tonnage (Sanchez, Jézégou à par.).

44. À l'exception des lingots de plomb hispaniques, découverts par Luc Long sur SM35, d'autres épaves pourraient renfermer des métaux en provenance d'Hispanie.

45. À partir des structures portuaires de l'anse des Laurons, il serait nécessaire de calculer le tirant d'eau maximal pour vérifier cette possibilité. l'accessibilité du canal et/ou de la mise en place de l'organisation du halage (en vert sur la Fig. 12).

\section{LES NAVIRES À CARGAISON MIXTE NON APTES À FRANCHIR LES BARRES DU RHÔNE}

En provenance d'Hispalis ou de Narbonne, ces navires pouvaient transporter des cargaisons mixtes avec des métaux et des amphores. Les navires devaient stationner à Fos et transborder dans des allèges les matières premières en direction de l'ostium metapinum, et les amphores en direction des deux autres embouchures (Fig. 13). L'étape du transbordement des amphores sur des allèges pouvait succéder à une période de stockage dans des horrea que l'on situerait à Fos. Deux remarques doivent être formulées :

- L'épave Plage d'Arles 4, chargée de lingots de cuivre sur lesquels repose la cargaison principale composée de 2000 amphores de Bétique, correspond parfaitement à cette configuration. Ce bateau pouvait alors distribuer à Fos les lingots de cuivre dans de petites unités en direction du Rhône Saint-Ferréol.

- L'hypothèse de bateaux à cargaison mixte en capacité de franchir les barres du Rhône, qui serait en contradiction avec le postulat de départ, n’a pas été retenue.

\section{CONCLUSION}

La concentration exceptionnelle d'épaves au large des SaintesMaries-de-la-Mer a conduit les chercheurs à focaliser leur attention uniquement sur le Rhône Saint-Ferréol. Mais si aujourd'hui cette ancienne embouchure a pu bénéficier du travail colossal de prospections sous-marines menées par Luc Long, les autres embouchures sont en pleine terre. D'un point de vue géomorphologique les deux autres bras étaient actifs (le Rhône de Daladel et le Rhône d'Ulmet) (Vella et al. 2016), mais il est en revanche plus difficile de savoir s'ils étaient navigables ou pas (Djaoui à par. 3).

Néanmoins, après plus d'une trentaine d'années de prospections, une spécificité commence à apparaître. En dépit des millions d'amphores des $\mathrm{I}^{\mathrm{er}}$ et $\mathrm{II}^{\mathrm{e}} \mathrm{S}$. qui ont de toute évidence emprunté la vallée du Rhône, aucune épave chargée d'amphores du HautEmpire n'est attestée devant l'embouchure du Rhône Saint-Ferréol.

L'hypothèse que le Rhône Saint-Ferréol était plus particulièrement dévolu aux matières premières et que les navires chargés d'amphores devaient croiser au large pour éviter la Camargue (Djaoui à par. 3) m'a conduit à envisager qu'au moins l'un des deux autres bras du Rhône, que ce soit celui de Daladel ou que ce soit celui d'Ulmet, devait être non seulement navigable mais également réservé aux amphores. Il s'agirait donc d'un trafic régulé en fonction des différentes embouchures du delta rhodanien et réparti entre produits comestibles et non comestibles.

Si l'on exclut la pérennité de lobes alluviaux émergents et donc d'une zone de mouillage située face à l'embouchure du Rhône Saint-Ferréol, on privilégiera l'hypothèse d'un port type salos situé au large de cette embouchure. Ce type de port, que l'on peut définir comme un «port en eau profonde ${ }^{46}$, présente en Camargue l'avantage d'éloigner les bateaux du delta tout en assurant un bon mouillage. Aussi, je propose de situer la zone de rupture de charge des

46. Expression de Pascal Arnaud que je remercie pour ses précieux conseils. 


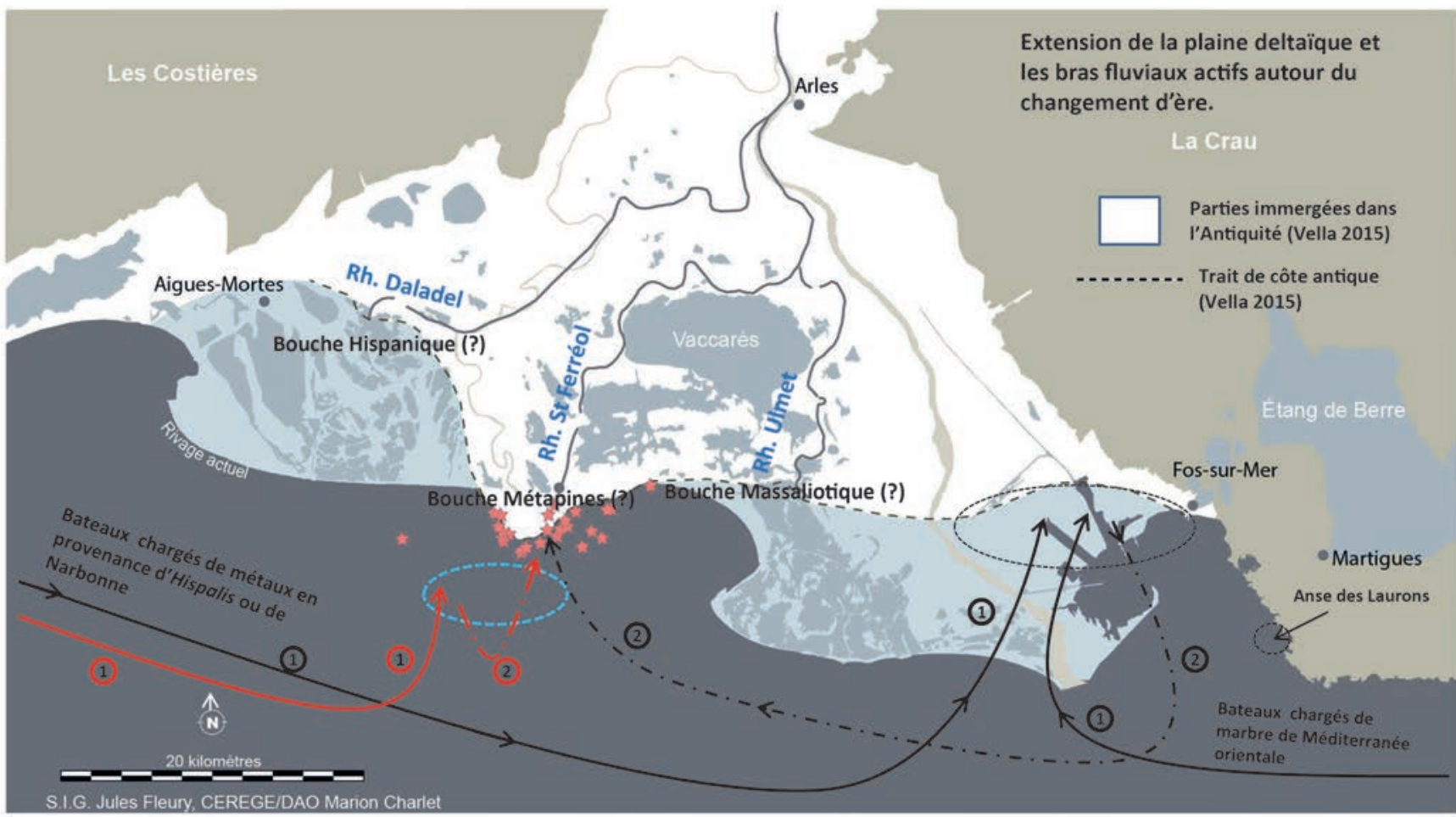

* Epaves antiques des Saintes-Maries-de-la-Mer ( Long 2004).

(-...) Port abrité ( limen)

Port en eau profonde (salos)

$\left\{\begin{array}{l}\stackrel{\text { (1) }}{\rightarrow} \text { Trajets des bateaux maritimes vers Fos } \\ \stackrel{\text { (2) }}{\rightarrow} \text { Trajet des allèges après transbordement à Fos }\end{array}\right.$

Fig. 9: Schéma commercial des bateaux chargés de matières premières en capacité de franchir les barres du Rhône (er S. apr. J.-C.) (D.A.O. : D. Djaoui).

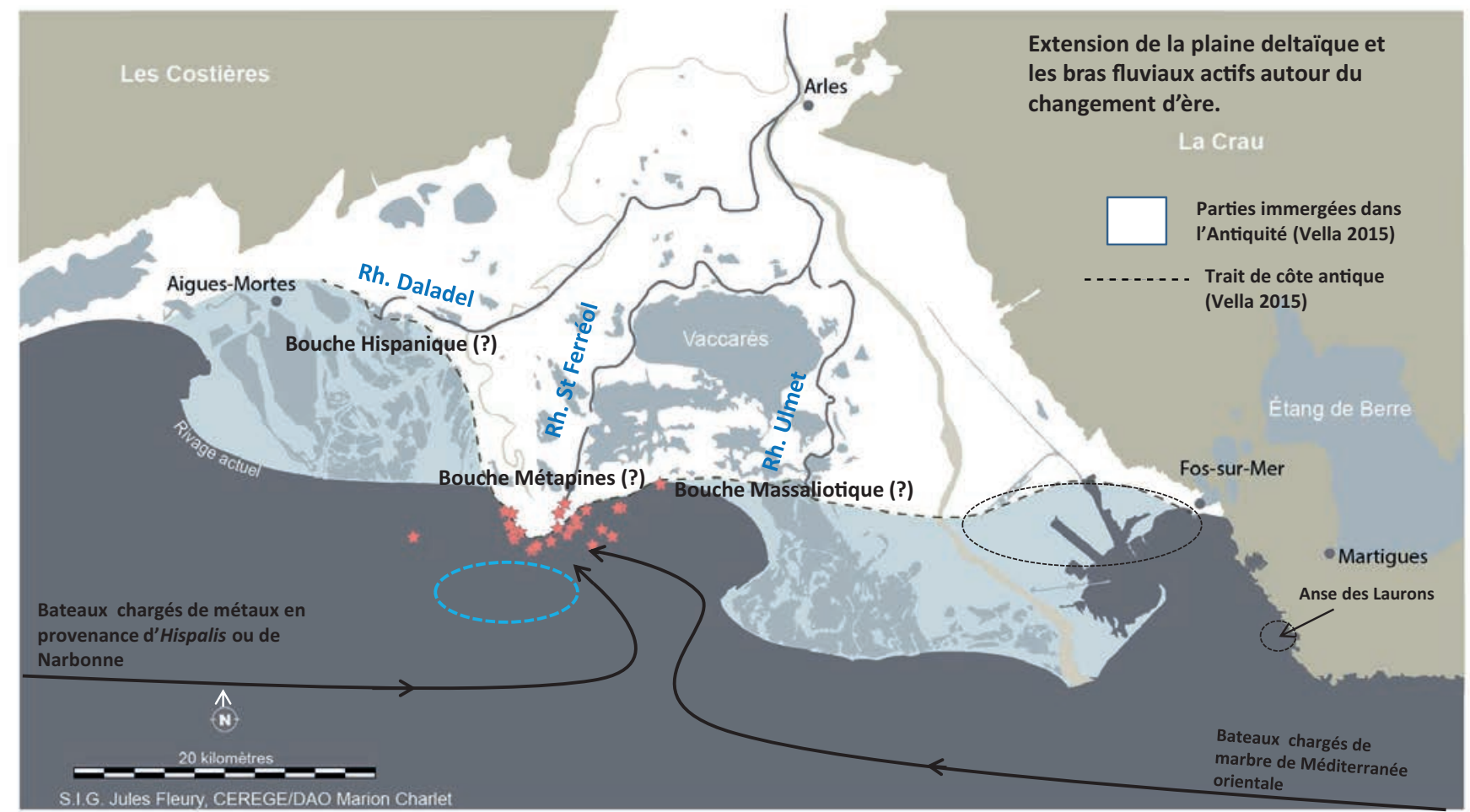

t Epaves antiques des Saintes-Maries-de-la-Mer ( Long 2004)

$\longrightarrow$ Trajets directs par vent favorable

(........ Port abrité ( limen) et/ou zone d'attente en cas de vent défavorable

Port en eau profonde (salos) et/ou zone d'attente en cas de vent défavorable

Fig. 10: Schéma commercial des bateaux maritimes chargés de matières premières dont le tirant d'eau ne permettait pas de franchir les barres du Rhône (ler S. apr. J.-C.) (D.A.O. : D. Djaoui). 


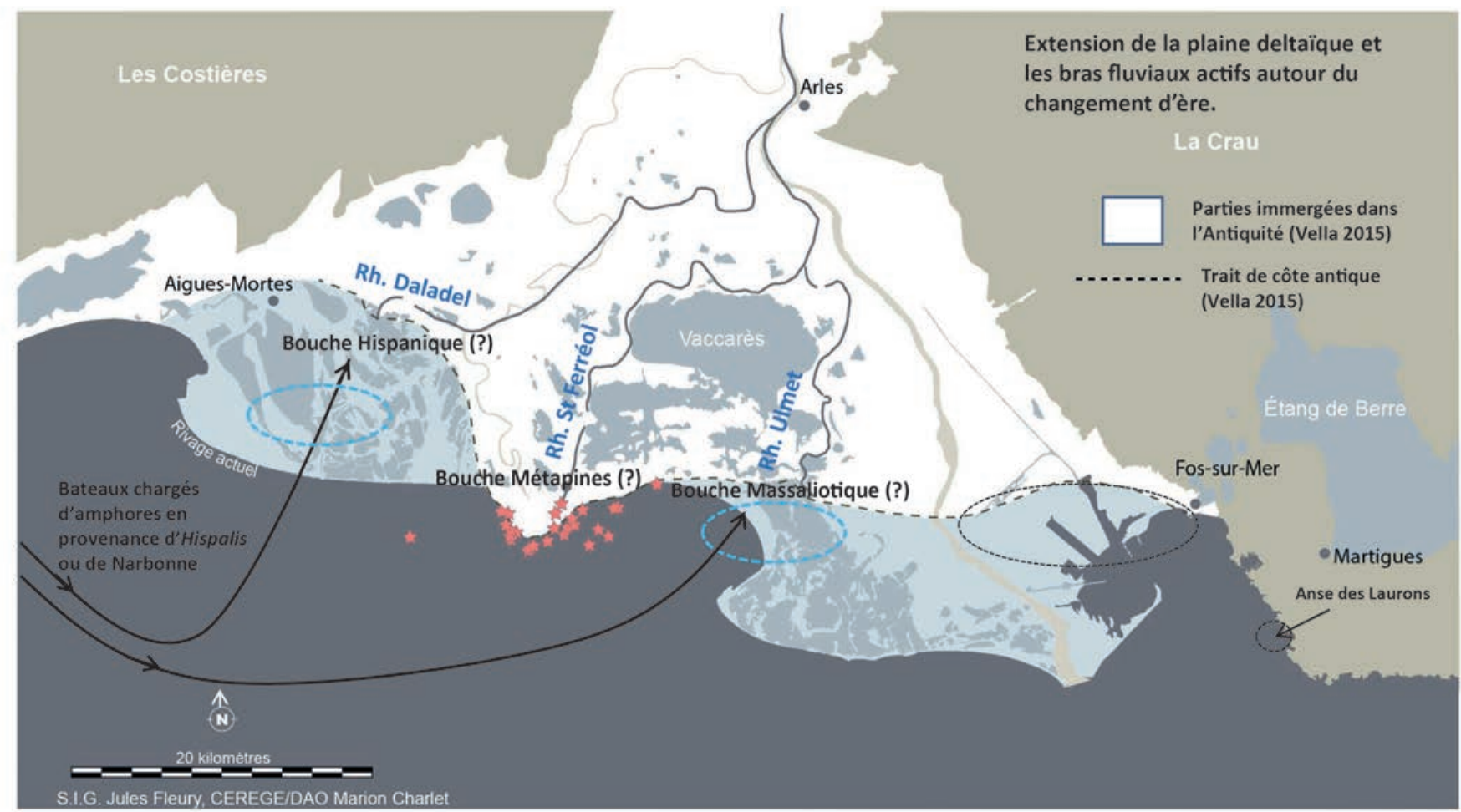

* Epaves antiques des Saintes-Maries-de-la-Mer ( Long 2004).

Port abrité ( limen) et/ou zone d'attente en cas de vent défavorable

$\longrightarrow$ Trajets directs par vent favorable

Port en eau profonde (salos) et/ou zone d'attente en cas de vent défavorable

Fig. 11: Schéma commercial des bateaux amphoriques en capacité de franchir les barres du Rhône (er S. apr. J.-C.) (D.A.O.: D. Djaoui).

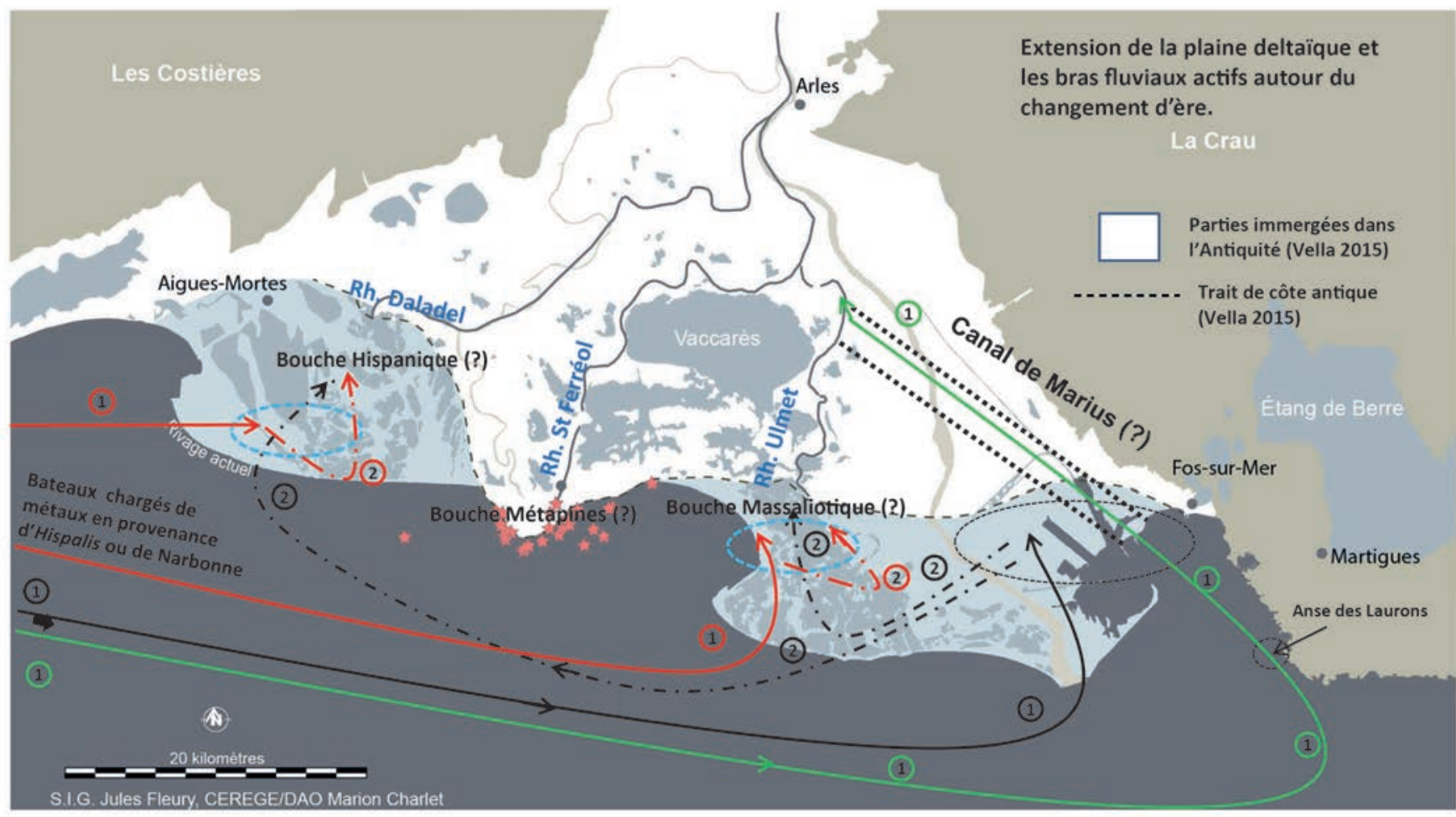

* Epaves antiques des Saintes-Maries-de-la-Mer ( Long 2004).

$\left\{\begin{array}{l}\stackrel{(1)}{\longrightarrow} \text { Trajets des bateaux maritimes vers Fos } \\ \stackrel{-}{\rightarrow} \text { Trajet des allèges après transbordement à Fos }\end{array}\right.$

Port abrité ( limen)

Port en eau profonde (salos)

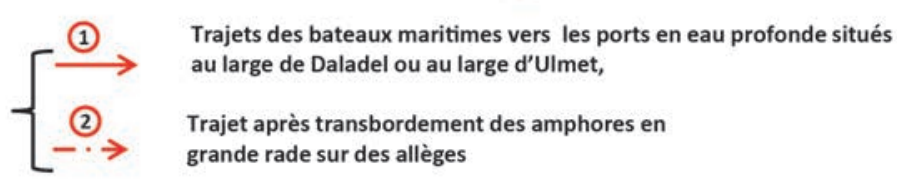

Fig. 12: Schéma commercial des bateaux amphoriques dont le tirant d'eau ne permettait pas de franchir les barres du Rhône (l丷r S. apr. J.-C.) (D.A.O.: D. Djaoui). 


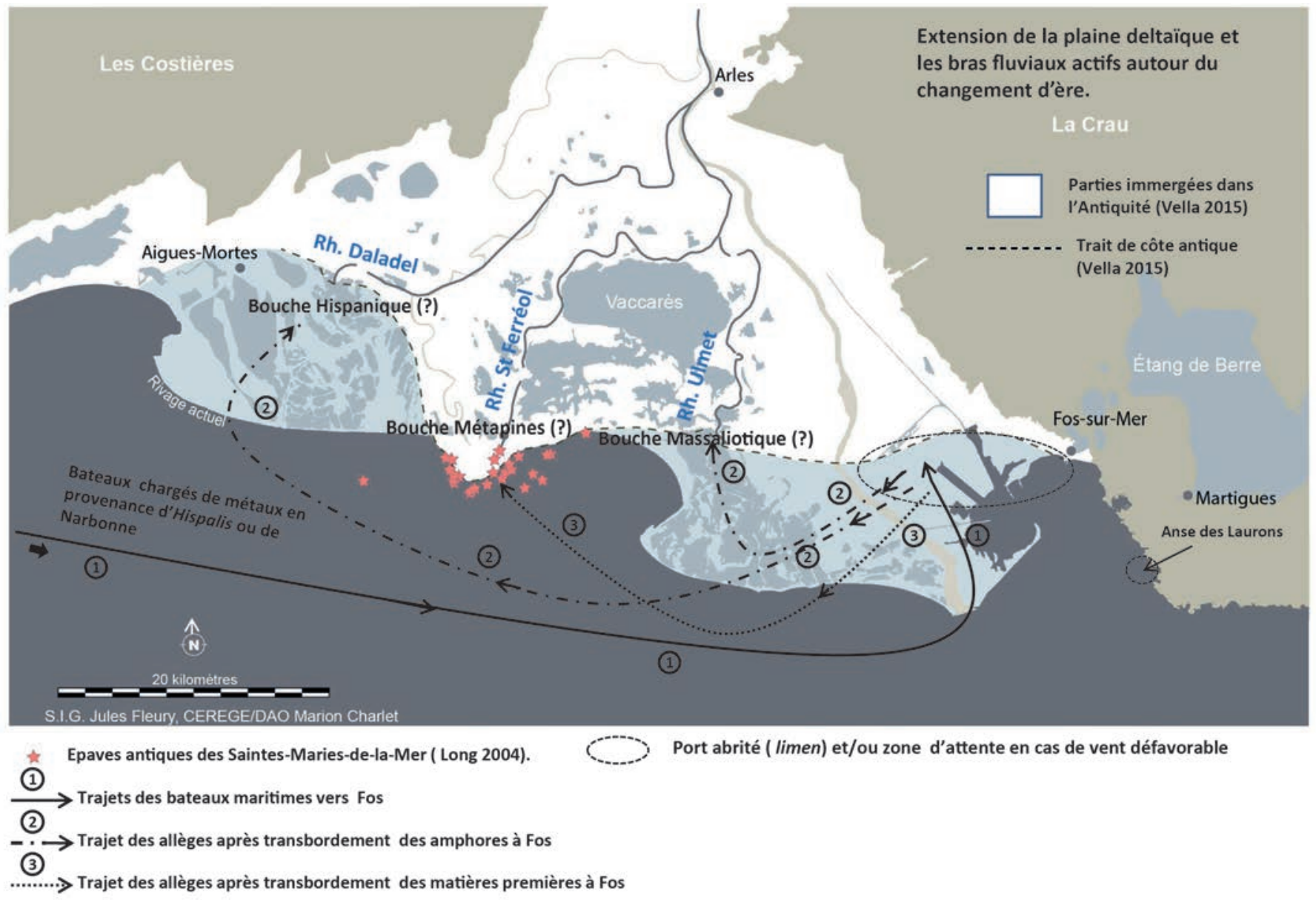

Fig. 13: Schéma commercial des bateaux à cargaison mixte dont le tirant d'eau ne permettait pas de franchir les barres du Rhône (Ir S. apr. J.-C.) (D.A.O.: D. Djaoui).

bateaux - qui n'avaient pas la capacité de remonter le Rhône - plus au large, et suivant un scénario comparable à celui de Strabon pour Ostie, c'est-à-dire avec «d'innombrables barques à rame qui allègent les cargaisons ». Dans cette hypothèse, la découverte des amphores situées à l'entrée du chenal de navigation du Rhône Saint-Ferréol (zone SM0) illustrerait davantage les aléas d'un trafic régulier qu'une véritable zone de rupture de charge.

Ce constat suppose l'existence d'autres ports, c'est-à-dire d'autres zones de transbordement des marchandises pour les bateaux maritimes les plus imposants, ou encore d'autres zones de mouillage pour les fluviomaritimes qui pouvaient attendre les vents favorables durant plusieurs semaines ${ }^{47}$.

$\mathrm{Au}$ sein de cette hiérarchie portuaire, la notion d'un unique avant-port maritime d'Arles n'a plus de sens et je rejoins la prudence de Pascal Arnaud en évitant l'utilisation du mot «avantport», qui demeure une notion mal définie pour l'Antiquité (Arnaud 2016, p. 140), au profit «des ports d'Arles»: les uns situés au large de l'embouchure du Rhône Saint-Ferréol, et vraisemblablement au large de celles de Daladel et/ou d'Ulmet, et les autres au sein du complexe portuaire de Fos. La spécialisation des embouchures du Rhône laisserait enfin envisager un ou plusieurs ports situés sur la section inférieure du Rhône Saint-Ferréol qui seraient en charge d'absorber les matières premières pour en assurer tant le contrôle,

47. Pour entrer dans le delta, il était indispensable de disposer de vents de secteur sud à est (Rigaud 2004, p. 272; Payn-Echalier 2010, p. 36). le stockage que la redistribution. Seules les recherches à venir permettront de confirmer ou démentir cette hypothèse ${ }^{48}$.

Le modèle proposé suppose une circulation de l'information du fonctionnement du complexe portuaire d'Arles et de ses ports à l'échelle de la Méditerranée. Jean Andreau explique que les réseaux d'information à distance appartiennent à trois catégories: l'État, l'élite et le commerce, pour lequel les ports constituent le lieu privilégié pour la diffusion auprès des marchands (Andreau 2002, p. 7-9), et donc des équipages. Aussi, il ne fait pas de doute que le mode de fonctionnement d'une telle logistique, impliquant la spécialisation des différentes embouchures, pouvait facilement être transmis à partir des différents ports de l'Empire. Informations qui, dans tous les cas, devaient circuler pour rendre compte aussi bien des mouvements incessants des barres d'avant côte que de la fluctuation des limitations de hauteur d'eau des embouchures ${ }^{49}$, ou

48. Cet article a bénéficié de nombreux relecteurs avec notamment l'équipe de Fos: Souen Fontaine, Mourad El Amouri et Frédéric Marty. Qu'ils en soient vivement remerciés. Un grand merci à Pascal Arnaud, Philippe Leveau, Philippe Rigaud, Claude Vella et Alain Charron pour leurs remarques et leurs précieux conseils bibliographiques. Je tiens tout particulièrement à remercier également Sabrina Marlier qui, outre ses conseils et corrections, m'a livré de précieuses informations encore inédites. Ce travail n'aurait enfin pas pu atteindre son but sans l'avis critique et bienveillant de Patrice Pomey qui a contribué à enrichir considérablement les arguments que je soutenais.

49. Des petites barques auxiliaires, ou scaphae, animées par des rameurs, pouvaient également stationner à l'entrée des embouchures pour orienter les bateaux à travers le dédale des barres d'avant-côte (Boetto 2006, p. 398-399). 
encore pour anticiper la logistique du halage. Dans ce nouveau schéma, la thèse de P. Horden et N. Purcell, selon laquelle la mer Méditerranée se caractérise par sa «connectivity» (Horden, Purcell 2000), reste plus que jamais d'actualité. Que ce soit par l'imbrication des différents ports entre eux, en eau profonde (salos), ou en zones protégées (limen), ou encore par la transmission de l'information des embouchures spécialisées au niveau de l'ensemble de la Méditerranée nord-occidentale.

Archéologue territorial

Musée départemental Arles antique Chercheur associé sous convention au Centre Camille Jullian, Aix Marseille Univ, CNRS, Minist Culture, CCJ, Aix-en-Provence, France david.djaouimdaa@gmail.com

\section{BIBLIOGRAPHIE}

ANDREAU J.

1987 L'espace de la vie financière à Rome, dans L'Urbs: espace urbain et

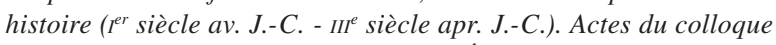
international de Rome, 1985, Rome, École Française de Rome (Publications de l'École française de Rome, 98).

2002 Présentation, dans J. Andreau, C. Virlouvet (dir.), L'information et la mer dans le monde romain, Rome, École Française de Rome (Collection de l'École française de Rome, 297), p. 1-17.

ARnAud P.

2010 Systèmes et hiérarchies portuaires en Narbonnaise, dans X. Delestre, $\mathrm{H}$. Marchesi (dir.), Archéologie des rivages méditerranéens, 50 ans de recherche, Actes du colloque d'Arles, 28-30 oct. 2009, Paris, Errance, p. 107-113.

2016 Entre mer et rivière: les ports fluviomaritimes de Méditerranée ancienne. Modèles et solutions, dans C. Sanchez, M.-P. Jézégou (dir.) 2016, p. 139-155.

Arnaud-Fassetta G.

2004 Le rôle du fleuve: les formations alluviales et la variation du risque fluvial depuis 5000 ans, dans C. Landuré, M. Pasqualini (dir.) 2004, p. 65-77.

BENOIT F.

1952 L'archéologie sous-marine en Provence, Revue des Études Ligures, 18.3-4, p. 237-307.

Boetto G.

2006 Les navires de Fiumicino (Italie): architecture, matériaux, types et fonctions. Contribution à l'étude du système portuaire de Rome à l'époque impériale, Thèse de doctorat soutenue à l'Université d'Aix-Marseille I, sous la direction de P. Pomey, non publiée.

BonNEFOUX DE P-M-J.

1855 Dictionnaire de la marine à voile, Paris, rééd. René Baudouin, 1980.

CONCHON C.

2007 Circulation fluviale et aménagements portuaires en France au XVIII ${ }^{e}$ siècle, dans L. Dumond, S. Durand, J. Thomas, Les ports dans l'Europe Méditerranéenne, Trafics et circulation, Images et représentations, XVI ${ }^{e}-X X I^{e}$ siècles, Actes du colloque tenu à Montpellier, 19-20 mars 2004, Montpellier, Presses Universitaires de la Méditerranée, p. 43-54.

DJaOui D.

2016 Les céramiques hispaniques du dépotoir portuaire d'Arles-Rhône 3 (50-140 ap. J.-C.), dans Actes III Congresso internacional de la SECAH, "Amphorae ex Hispania. Paisajes de producción y de consumo», Tarragone, 10-13 dec. 2014, Tarragone, SECAH (Monografias Ex officina Hispana, III), p. 962-975.

À paraître 1 Difficultés et intérêts à définir un faciès portuaire fluviomaritime en zone péri-urbaine (50-140 ap. J.-C.): fouilles subaquatiques à Arles (Bouches-du-Rhône), dans P. Ballet, S. Lemaître, I. Bertrand (dir.), Les mobiliers archéologiques dans leur contexte, de la Gaule à l'Orient méditerranéen, Colloque international de Poitiers, 27-29 octobre 2014, Rennes, PUR.
À paraître 2 Le transport en vrac: dolia ou tonneaux? dans Nouvelles recherches sur les dolia. L'exemple de la Méditerranée nord-occidentale à l'époque romaine ( $t^{e r}$ s. av. J.-C. $-I I I^{e}$ s.. ap. J.-C.), Actes de la table ronde des 26 et 27 septembre 2013, Aspiran.

À paraître $3 \mathrm{La}$ circulation des matières premières et des amphores à l'échelle du delta du Rhône au Haut-Empire, dans D. Boisseuil, C. Rico (dir.), Marché des matières premières dans l'Antiquité et au Moyen Âge.

Djaoui D., Capelli C.

À paraître, Objets d'importation ou objets personnels? La dotation de bord des marins au regard du grand commerce, l'exemple du dépotoir portuaire d'Arles-Rhône 3, dans Actes du colloque de la SFECAG de Narbonne, 25-28 mai 2017.

DJaOui D., PIQuìs G., BotTe E.

2014 Nouvelles données sur les pots dits «à garum» du Latium, d'après les découvertes subaquatiques du Rhône (Arles), dans E. Botte, V. Leitch (dir.), Fish \& Ships, Production and commerce of salsamenta during Antiquity, Actes de l'atelier doctoral à Rome, 18-22 juin 2012, Arles, Errance/Aix-en-Provence, Centre Camille Jullian (BIAMA, 17), p. 175-196.

EHMig U.

2014 Publicité ou conséquences des risques du transport maritime? Sur la fonction des tituli picti des amphores romaines dans le contexte des prêts maritimes, Cahiers du Centre Gustave Glotz, XXV, p. 89-106.

El Amouri M., Fontaine S., Marty F.

2015 Un port maritime d'Arles à l'époque romaine à Fos-sur-Mer, dans C. Landuré, C. Vella, M. Charlet (dir.) 2015, p. 134-136.

Fontaine S., El Amouri M., Marty F., Rousse C.

À paraître The Roman harbour system of Fossae Marianae (Gulf of Fos, South of France): New data on the submerged structures and main tracks of a new multidisciplinary research project, dans Harbours from the Roman Period to the Middle Ages - Harbours as objects of interdisciplinary research: Archaeology, History, Geosciences, Museum at the Institut of Geosciences, 30 sept.-3 oct. 2015, Kiel, Allemagne.

\section{Gambarini B., Chiesa A.}

1746 Delle cagioni e de' rimedi delle inondazioni del Tevere. Della somma difficoltà d'introdurre una felice e stabile navigazione da Ponte Nuovo sotto Perugia fino alla foce della Nera nel Tevere, e del modo di renderlo navigabile dentro Roma, Rome.

Gassend J.-M., Liou B., Ximénès S.

1984 L'épave 2 de l'anse des Laurons, Archaeonautica, 4, p. 75-105.

Gassend J.-M., Maillet B.

2004 Structures immergées dans l'anse Saint Gervais (Fos-sur-Mer, Bouches-du-Rhône), dans C. Landuré, M. Pasqualini (dir.) 2004, p. 151-163.

Gateau F.

1996 L'étang-de-Berre, Paris, Académie des Inscriptions et Belles-Lettres (CAG 13/1). 
Giacobbi-Lequément M.-F

1997 La céramique de l'épave Fos 1, Archaeonautica, 7, p. 167-191.

GoIrand J.-P. et alii

2016 J.-P. Goirand, F. Salomon, C. Vittori, G. Boetto, E. Pleuger, J. Christiansen, B. Noirot, A. Pellegrino, I. Mazzini, L. Sadori, C. Oberlin, C. Pepe, V. Ruscito, P. Arnaud, Géoarchéologie du bassin portuaire d'Ostie, dans Sanchez, Jézégou (dir.) 2016, p. 305-319.

Horden P., PURCELl N.

2000 The Corrupting Sea. A Study of Mediterranean History, A Study of Mediterranean History, Oxford, Blackwell Publishing.

JÉZÉGOU M.-P.

1998 Le mobilier de l'épave Saint-Gervais 2 (vII ${ }^{\mathrm{e}}$ s.) à Fos-sur-Mer (BDR), dans M. Bonifay, M.-B. Carre, Y. Rigoir, Fouilles à Marseille, Les mobiliers ( $I^{e r}-V I I^{e}$ siècles ap. J.-C.), Paris/Lattes, Errance/A.D.A.M. (Études Massaliètes, 5), p. 343-351.

KeAY S.

2012 The Port system of imperial Rome, dans S. Keay (dir.) Rome, Portus and the Mediterranean, London, the British School at Rome (Archaeological Monographs of the British School at Rome, 21), p. 33-67.

Landuré C., Pasqualini M. (Dir.)

2004 Delta du Rhône. Camargue antique, médiévale et moderne, Gap, Éditions de l'Association Provence Archéologie (Bulletin Archéologique de Provence, Supplément 2).

Landuré C., Vella C., Charlet M. (Dir.)

2015 La Camargue. Au détour d'un méandre. Études archéologiques \& environnementales du Rhône d'Ulmet, Catalogue d'exposition, Musée départemental Arles antique, Arles, CD13/MdAa.

LEGENDRE $\mathrm{S}$.

2015 Les sigillées italiques du golfe de Fos, Master 2 (MoMARCH), sous la direction de J.-Chr. Sourisseau, Université Aix-Marseille, non publié.

LIMA C.

2002 Les «ancres» en pierre issues des fouilles antiques de la place Jules-Vernes, à Marseille, essai typologique et d'interprétation du matériel lithique, Mémoire de DEA, Université de Provence, sous la direction de Patrice Pomey, non publié.

Liou B., Sciallano M.

1987 Le trafic du port de Fos dans l'Antiquité: essai d'évaluation à partir des amphores, dans Actes du colloque de la SFECAG de Lezoux, 4-7 mai 1987, Marseille, SFECAG, p. 153-157.

LONG L.

1997 Inventaire des épaves de Camargue, de l'Espiguette au Grand Rhône. Des cargaisons de fer antiques aux gisements du XIX ${ }^{e}$ s. Leur contribution à l'étude du paléorivage, dans M. Baudat (dir.), Crau, Alpilles, Camargue. Histoire et archéologie, Actes du colloque d'Arles, 18-19 Nov. 1995, Arles, Groupe Archéologique Arlésien, p. 59-115.

2008 Secrets du Rhône. Les trésors archéologiques du fleuve à Arles, Arles, Actes Sud.

2009 «Duplex Arelas», fluviale et maritime, dans F. Dumasy, F. Queyrel (dir.), Archéologie et environnement dans la Méditerranée antique, Genève, Droz (Ecole Pratique des Hautes Études, III; Sciences historiques et philologiques, 42), p. 195-220.

LONG L., DUPERron G.

2011a Bouches-du-Rhône. Au large des Saintes-Maries-de-la-Mer, dans Bilan Scientifique du Drassm 2011, Paris, Ministère de la culture et de la communication, p. 56-61.

2011b Recherches sous-marines au large des Saintes-Maries-de-la-Mer (Bouches-du-Rhône), Sur les traces de l'avant-port maritime d'Arles, dans Actes du colloque de la SFECAG d'Arles, 2-5 juin 2011, Marseille, SFECAG, p. 97-118.

2013 Navigation et commerce dans le delta du Rhône: l'épave ArlesRhône 14 (III ${ }^{\mathrm{e}}$ S. apr. J.-C.), dans S. Mauné, G. Duperron éd., Du
Rhône aux Pyrénées, Aspects de la vie matérielle en Gaule Narbonnaise II, Montagnac, ed. M. Mergoil (Archéologie et Histoire Romaine, 25), p. 125-167.

2016 Navigation et commerce dans le delta du Rhône durant l'Antiquité : bilan des recherches sur le port fluvial d'Arles et ses avant-ports maritimes, dans C. Sanchez, M.-P. Jézégou (dir.) 2016, p. 199-217.

LONG L., ILLOUZE A.

2004 Nouvelles épaves de Camargue. Les gisements antiques, modernes et contemporains, Delta du Rhône, dans C. Landuré, M. Pasqualini (dir.) 2004, p. 291-330.

Long L., PiCARd P. (DIR.)

2009 César. Le Rhône pour mémoire. Vingt ans de fouilles dans le fleuve à Arles, catalogue d'exposition, Arles, Actes Sud, Musée départemental Arles antique.

Long L., Piton J., DJaOui D.

2009 Les céramiques communes des gisements du Rhône à Arles, le faciès portuaire d'époque impériale, dans M. Pasqualini (dir.), Les céramiques communes d'Italie et de Narbonnaise, Structures de production, typologies et contextes inédits (II' S. av. J.-C. - III ${ }^{e}$ S. apr. J.-C.), Actes de la table ronde de Naples (2-3 novembre 2006), Naples, Centre Jean Bérand (coll. CJB, 30), p. 569-614.

MARLIER S.

2008 Architecture et espace de navigation des navires à dolia, Archaeonautica, 15, p. 153-173.

À paraître Navires et navigation dans le delta du Rhône à l'époque romaine.

Marty F., ZaAraoui Y.

2009 Contextes céramiques du Haut-Empire de la bonification de l'Estagnon à Fos-sur-Mer, dans Actes du congrès de la SFECAG de Colmar, 21-24 mai 2009, Marseille, SFECAG, p. 397-426.

Mellinand Ph., Sivan O.

2011 Gare maritime, Arles, Rapport d'opération, Diagnostic archéologique, Inrap Méditerranée, Nîmes.

\section{Moerman M.}

1993 Le port romain des Laurons (Martigues), La villa et les structures terrestres, Thèse de doctorat soutenue à l'Université de Provence, sous la direction d'André Tchernia, non publiée.

\section{Payn-Echalier P.}

2010 Entre fleuve et mer, le port d'Arles et le delta du Rhône (XvI ${ }^{\mathrm{e}}$ $\mathrm{XVIII}{ }^{\mathrm{e}} \mathrm{s}$.), Rives méditerranéennes, 35, p. 29-44.

Payn-Echalier P., Rigaud P.

2016 Pierre Giot, un capitaine marin arlésien «dans la tourmente», Journal, livre de bord, correspondance (1792-1816), Aix-en Provence, Presses Universitaires de Provence (Le Temps de l'Histoire).

POMEY P. (DIR.)

1987-1988 Recherches sous-marines, Gallia Informations. Préhistoire et Histoire 1987-1988-1, p. 1-78.

1992 Recherches sous-marines, Gallia Informations. Préhistoire et Histoire 1992-1, p. 1-85.

1997 La navigation dans l'Antiquité, Aix-en-Provence, Édisud.

Pomey P., Gianfrotta P. A.

1981 L'archéologie sous la mer, Paris, Nathan.

Rey T., Lefevre D., Vella C.

2009 Deltaic plain development and environmental changes in the Petite Camargue, Rhone Delta, France, in the past 2000 years, Quaternary Research, 71, p. 284-294.

\section{Rigaud Ph.}

2004 La navigation dans le delta, dans Landuré, Pasqualini (dir.) 2004, p. 271-276. 
RosSIAUD J.

2007 Le Rhône au Moyen Âge: histoire et représentations d'un fleuve, Paris, Aubier (Collection historique).

Rougé J.

1966 Recherches sur l'organisation du commerce maritime en Méditerranée sous l'Empire romain, Paris, S.E.V.P.E.N.

1987 Routes et ports de la Méditerranée antique, Revue d'Études Ligures, 53 , p. $151-170$.

Sabatier F., Kulling B.

À paraître Caractérisation et dynamique de la charge de fond à l'embouchure du Grand Rhône. Synthèse, dans Rapport pour l'Observatoire des Sédiments du Rhône, Aix-Marseille Université.

SANCHEZ C., JÉZÉGOU M.-P. (DIR.)

2016 Les ports dans l'espace méditerranéen antique, Narbonne et les systèmes portuaires fluvio-lagunaires, Actes du colloque international, Montpellier, 22 et 24 mai 2014, Montpellier-Lattes, Ed. de l'Association de la RAN (RAN, suppl. 44).

SANCHEZ C., JÉZÉGOU M.-P.

À paraître Un complejo portuario romano descubierto en las albuferas narbonenses, dans I. Negueruela, R. Castillo, P. Recio (dir.), Proceedings of the International Conference on Underwater Archaeology IKUWA V (Cartagena, 15-19 de octubre 2014), Ministerio de Educación, Cultura y Deporte, ARQUA.

SCIALlano M., LeFFy R.

1992 Bouches-du-Rhône. Au large de Fos-sur-Mer, dans Bilan Scientifique du DRASM 1992, Paris, Ministère de l'Education nationale et de la Culture, p. 42.
TCHernia A, Viviers D.

2000

Athènes, Rome et leurs avant-ports: "mégapoles» antiques et trafics méditerranéens, dans C. Nicolet (Dir.), Mégapoles méditerranéennes; Géographie urbaine rétrospective, Actes du colloque de l'EFR et de la MMSH, 1996, Rome, École Française de Rome, p. $761-801$

TRÉZINY H.

2004 Sources écrites grecques et latines, dans C. Landuré, M. Pasqualini (dir.) 2004, p. 93-104

Vella C. et alii

2005 C. Vella, T-J. Fleury, G. Raccasi, M. Provansal, F. Sabatier, M. Bourcier, Evolution of the Rhône delta plain in the Holocene, Marine Geology, 222-223, p. 235-265.

2016 C. Vella, C. Landuré, L. Long, Ph. Dussouillez, J. Fleury, C. Tomatis, O. Sivan, F. Marty, D. Isoardi, V. Pothin, Ports fluviaux, ports lagunaires du Rhône et son delta à l'Antiquité: mobilité environnementale et dynamiques géomorphologiques comme contraintes à l'aménagement (Port d'Arles, Fos/Saint-Gervais, Ulmet), dans C. Sanchez et M.-P. Jézégou 2016 (dir.), p. 353-368.

\section{Ximénès S., Moerman M.}

1987 Les épaves I, III et IV du port romain de l'anse des Laurons (Commune de Martigues, Bouches du Rhône), Cahiers d'Archéologie Subaquatique, VI, p. 171-182.

1988 L'anse des Laurons: structures portuaires, Cahiers d'Archéologie Subaquatique, VII, p. 121-129. 